\title{
Integrated modeling of insect flight: from morphology, kinematics to aerodynamics
}

\author{
Hao, Liu \\ (Received 24 December 2007; Published xx xx 2008)
}

\author{
Manuscript correspondence: \\ Dr. Hao Liu \\ Professor of Biomechanical Engineering \\ Graduate School of Engineering \\ Chiba University \\ 1-33 Yayoi-cho, Inage-ku, Chiba 263-8522, JAPAN \\ Phone/Fax: +81-43-290-3228 \\ Email: hliu@faculty.chiba-u.jp
}




\begin{abstract}
An integrated and rigorous model for the simulation of insect flapping flight is addressed. The method is very versatile, easily integrating the modeling of realistic wing-body morphology, realistic flapping-wing and body kinematics, and unsteady aerodynamics in insect flight. A morphological model is built based on an effective differential geometric method for reconstructing geometry of and a specific grid generator for the wings and body; and a kinematic model is constructed capable to mimic the realistic wing-body kinematics of flapping flight. A fortified FVM-based NS solver for dynamically moving multi-blocked, overset-grid systems is developed and verified to be self-consistent by a variety of benchmark tests; and evaluation of flapping energetics is established on inertial and aerodynamic forces, torques and powers. Validation of this integrated insect dynamic flight simulator is achieved by comparisons of aerodynamic force-production with measurements in terms of the time-varying and mean lift and drag forces. Results for three typical insect hovering flights (hawkmoth, honeybee and fruitfly) over a wide rang of Reynolds numbers from $O\left(10^{2}\right)$ to $O\left(10^{4}\right)$ demonstrate its feasibility in accurately modeling and quantitatively evaluating the unsteady aerodynamic mechanisms in insect flapping flight.
\end{abstract}

Keywords: insect flight, hovering, morphology, kinematics, aerodynamics, Navier-Stokes equations, multi-block, overset grid, vortex dynamics 


\section{Introduction}

\section{A. Overview of insect flight}

Flying insects, in general, perform flapping wing flight because they have to create not only lift to stay airborne but also thrust for forward and darting flight, in achieving remarkable maneuvers with rapid accelerations and decelerations. Aerodynamics associated with the insect flapping wing flight prominently features unsteady motions at moderate Reynolds numbers ranging over $O\left(10^{1}\right)$ to $O\left(10^{4}\right)$, which is normally characterized by large-scale vortex structures, complicated flapping wing kinematics and flexible-wing structures [1-4]. Thus a challenging problem in uncovering aerodynamic mechanisms in insect flight is to answer a central question of how the large-scale vortex dynamics and complicated wake topology are generated and how they correlate to the force production.

Studies $[4,6-11,13-14,18-19,21-26]$ on aerodynamics of a single flapping wing model have been the main subject till now; and a general conclusion is that insects keep them aloft by using a high-lift, unsteady aerodynamic mechanism based on the sophisticated vortices. Experimental studies using robotic insect wing models have made significant contributions in quantifying some of the unsteady aerodynamic effects including the leading edge vortex mechanisms, the wing rotation and the wake capture mechanisms and so forth $[7-8,11,13]$. Recent direct measurements of the vortical structures around real insects and birds [7-8, $11,13,15-17,21-25,27,29]$ further enhance our insights into quantitative description on such vortex dynamic-based aerodynamics in flapping wing flight. However, limited to specific insects of hawkmoth and fruitfly and to specific focus on the near-field flows around non-realistic hovering models $[7-8,11,13,16]$, or, tracking on the footprint of the vortex dynamics in a manner of the far-field flow fields around real animals $[15,17,26,28,30]$, a universal explanation on insect flapping wing aerodynamic mechanisms still remains controversial [13]. This leads to recognition that an integrative study is necessary for systematically and quantitatively investigating the effects of sizing or Reynolds number, morphology and kinematics on flapping wing flight.

\section{B. Integrated modeling of insect flight}

To tackle this difficult problem one needs to deal with unsteady aerodynamics about an insect-like multi-body system with consideration of dynamically oscillating boundaries of flapping wings and 
complicated wing-wing and wing-body interactions. While flows around flying insects obey the Navier-Stokes equation a comprehensive answer to the question depends upon multi scale solutions of the problem that integrate the vortex dynamics of all scales in terms of the near and far fields and that establish an essential three-dimensional mechanism of flapping wing aerodynamics. To reveal such an essential three-dimensional mechanism [31-32], a systematic and quantitative study of insect flapping wing flight is a must, which need to be established on the basis of realistic morphology and kinematics and is capable to quantify the vortex dynamics, the wake topology and their correlation with the force-production.

We here address an integrated and rigorous model for the simulation of insect flapping flight. The majority of the current insect-like model is the integrated modeling methodology from realistic body-wing morphology, the flapping-wing and body kinematics to the unsteady aerodynamics as well as the quantitative evaluation of flapping energetics on inertial and aerodynamic forces, torques and powers. Till now most CFD-based models employ non-realistic wing models in a fashion of single or paired wings in 2D or $3 \mathrm{D}[14,18-19,28]$ with a specific focus on how important the flapping motions influence the viscous vortex flows but with less attention to the modeling efficiency and the integrated correlation between the near-and far-field vortex dynamics and the force-production. Some studies focus on efficient algorithms development capable for treating arbitrary boundaries with complex geometries of wing and/or body [24, 27] by means of immersed boundary methods, etc in Cartesian coordinate systems but using some simple kinematic models. To date, no model has been reported which is versatile to easily construct a morphological model with realistic geometries of both wings and body, which is capable to accurately mimic complicated movements of realistic flapping wings and body kinematics, and which is able to efficiently solve the Navier-Stokes (NS) equations for a multi-blocked, overset-grid system with dynamically moving boundaries and meshes. In the following we demonstrate that the current model has such capability and the results of insect hovering flight can be obtained in a reasonable amount of time.

\section{Integrated modeling methodology}

\section{A brief overview of current model}

The current model integrates the modeling of morphology-kinematics-aerodynamics in insect flapping 
flight; and three typical insect hovering models covering a range of Res from $O\left(10^{2}\right)$ to $O\left(10^{4}\right)$ demonstrate its feasibility in efficiently and accurately modeling and evaluating the insect flapping flight. The morphological modeling is based on a differential geometric method for reconstructing wing-body geometry with each cross-section approximated as an ellipse, a specific structured-grid generator, and a multi-blocked overset grid system in dealing with complex wing-body geometries and complicated flapping movements. The kinematic modeling is capable to mimic realistic wing-body kinematics in terms of the body angle, the stroke plane angle, and the positional-feathering-elevation angles, which is combined with an analytical method for dynamic regridding. A fortified finite volume method (FVM)-based NS solver for the dynamically deforming multi-blocked, overset-grid system is developed and verified; and validation is implemented through an extensive study of aerodynamic force-production by comparison with experimental results in terms of the time varying and mean lift and drag forces. The computing performance is further confirmed by testing on a PC (Dell Precision 690) with DuralCore Intel(R) CPU(3.0GHz) and a memory of 4.0GB, which takes approximately $10 \mathrm{hrs}$ of CPU time for a hawkmoth-like wing-body model undergoing hovering for four complete beat cycle, with approx. 340000 grid points of two wing blocks and a body block.

\section{A. Coordinate systems in modeling of insect flapping flight}

We define three coordinate systems in modeling of insect flapping flight, as depicted in Fig. 1:1) a wing-fixed system $\left(x_{w}, y_{w}, z_{w}\right)$ with its origin at the pivot point of the right wing and 'flapping' with the flapping wing; 2) a body-fixed system $\left(x_{b}, y_{b}, z_{b}\right)$ with its origin at the center of mass of and moving together with the object; and 3) a global system $(X, Y, Z)$ as an inertial system being fixed horizontally. Body posture (Fig. 1(a)) is described by the stroke plane angle $\eta$ and the body angle $\chi$, relative to horizontal; the angles of pitch $\beta$, roll $\psi$, and yaw $\gamma$ with respect to the body-fixed system. Wing position parameters are defined (Fig. 1(b)) within the stroke plane: the wingtip path is indicated by the closed circle composed of a solid for downstroke and a dashed for upstroke, respectively; the positional angle $\phi$ is the angle between the $y_{s p}$ axis and the projection $y_{w}$, of the $y_{w}$ axis onto the stroke plane; the elevation angle $\theta$ describes the rotation about 
the axis $z_{w}$ between the wing axis $y_{w}$ and the stroke plane $y_{w}$, and the angle of attack $\alpha$ is defined as the feathering (rotation) of the wing axis $y_{w}$.

\section{B. Morphological modeling}

The morphological modeling is established on the basis of a differential geometric method [20] for reconstructing wing-body geometry by approximating each cross-section as an ellipse. It may be clarified in four-fold: 1) imaging to digitize 2D or 3D images in terms of pixel or voxel data; 2) segmentation to extract the object's shape in terms of a skeleton model; 3) surface fitting for the reconstructed model; 4) modeling and domain discretization to create the geometric model in 3D and to decompose the computational domain. One notable advantage of this methodology lies in a fact that it unifies the modeling of both morphology and kinematics, which enables an effective and non-time consuming process of simultaneous generation of both the wing-body geometric model and computational grids as well as of dynamic regridding.

\section{A differential geometry method}

Consider a space curve $\boldsymbol{L}$ (Fig. 2) that expresses a data string, $\boldsymbol{r}_{\boldsymbol{i}}=\left(x_{i}, y_{i}, z_{i}\right)$, where $\boldsymbol{r}_{\boldsymbol{i}}$ is a positional vector, and $(i, j, k)$ is a unit vector, which is extracted as a centerline of the object based on the digitized imaging process. This discrete space curve can be rewritten as a function of its arc length, such as:

$$
\boldsymbol{L}: \boldsymbol{r}=(x, y, z)=(x(s), y(s), z(s)),
$$

where $s$ denotes the arc length, which, if given a data string of $N$ points, can be calculated as $s_{i}=\operatorname{Sum}\left(d s_{i}\right)$, $d s_{i}=\left|r_{i}-r_{i-1}\right|, i=1,2, \ldots, i, \ldots N$.

Next, we calculate the curvature and torsion of the space curve $\boldsymbol{L}$ to quantify geometric changes at point $P_{i}$, and to get the tangent vector $\boldsymbol{T}$, the principal normal vector $\boldsymbol{N}$, and the binormal vector $\boldsymbol{B}$ as:

$$
\boldsymbol{T}=\frac{d \boldsymbol{r}}{d s}, \boldsymbol{N}=\frac{d \boldsymbol{T}}{d s} / \kappa, \boldsymbol{B}=\boldsymbol{T} \times \boldsymbol{N},
$$

where the curvature is defined as $\kappa=|d \boldsymbol{T} / d s|=|d \boldsymbol{r} / d s|$. Note that using the Frenet-Serret formula the binormal vector $\boldsymbol{B}$ can be expressed as a product of $\boldsymbol{T}$ and $\boldsymbol{N}$, and hence $d \boldsymbol{N} / d s=\tau \boldsymbol{B}-\boldsymbol{\kappa} \boldsymbol{T}$ and $d \boldsymbol{B} / d s=-\tau \boldsymbol{N}$, where the torsion $\tau$ may be defined as 


$$
\tau=\left[\begin{array}{ccc}
\frac{d x}{d s} & \frac{d y}{d s} & \frac{d z}{d s} \\
\frac{d^{2} x}{d s^{2}} & \frac{d^{2} y}{d s^{2}} & \frac{d^{2} z}{d s^{2}} \\
\frac{d^{3} x}{d s^{3}} & \frac{d^{3} y}{d s^{3}} & \frac{d^{3} z}{d s^{3}}
\end{array}\right] / \kappa^{2}
$$

Hence, given a data string $\boldsymbol{r}_{\boldsymbol{i}}$ one can reconstruct a space curve in terms of the arc length, and obtain the curvature and the torsion at any point on the curve by simply calculating those derivatives as in Eq. (2) and Eq. (3). Then, a space plane perpendicular to the tangential direction yields, which is determined by the three vectors of $\boldsymbol{T}, \boldsymbol{N}$ and $\boldsymbol{B}$.

To reconstruct a 3D geometry of the object, by approximating each cross-section as an ellipse we further introduce a data string for the two radii, namely, $\boldsymbol{R}=\boldsymbol{R}(s)$ or $\boldsymbol{R}_{i}=\boldsymbol{R}\left(s_{i}\right)$ in a discrete form, where $i=1,2,3, i, \ldots$ $N$. Now one only needs to map the local two-dimensional outline (the ellipse) onto the defined space plane $\boldsymbol{T N B}$. This is done through a straightforward transformation (Fig. 2(a)) by using unit vectors $\boldsymbol{e}_{T}, \boldsymbol{e}_{N}, \boldsymbol{e}_{\boldsymbol{B}}$ of the vectors $\boldsymbol{T}, \boldsymbol{N}$, and $\boldsymbol{B}$, in forms of,

$$
\left[\begin{array}{c}
e_{T} \\
e_{N} \\
e_{B}
\end{array}\right]=\left[\begin{array}{lll}
e_{T x} & e_{T y} & e_{T z} \\
e_{N x} & e_{N y} & e_{n z} \\
e_{B x} & e_{B y} & e_{B z}
\end{array}\right]\left[\begin{array}{l}
i \\
j \\
k
\end{array}\right] .
$$

Hence, a local positional vector $\boldsymbol{r}^{\prime}$ in the local coordinate system $(\zeta, \eta, \xi)$ on the space plane $\boldsymbol{T N B}$ and the corresponding positional vector $\boldsymbol{r}$ in the global coordinate system $(x, y, z)$ may be represented as:

$$
\begin{aligned}
& r^{\prime}=\xi e_{T}+\eta e_{N}+e_{B} \\
& =0 e_{T}+\eta_{2 D} e_{N}+\varsigma_{2 D} e_{B}=\left(\eta_{2 D} e_{N x}+\varsigma_{2 D} e_{B x}\right) i+\left(\eta_{2 D} e_{N y}+\varsigma_{2 D} e_{B y}\right) j+\left(\eta_{2 D} e_{N z}+\varsigma_{2 D} e_{B z}\right) k \\
& r=r_{i}+r^{\prime} \\
& =\left(x_{i}+\eta_{2 D} e_{N x}+\varsigma_{2 D} e_{B x}\right) i+\left(y_{i}+\eta_{2 D} e_{N y}+\varsigma_{2 D} e_{B y}\right) j+\left(z_{i}+\eta_{2 D} e_{N z}+\varsigma_{2 D} e_{B z}\right) k
\end{aligned}
$$

Note that a grid system in 2D, if generated for a circular cylinder, can be easily mapped to all the space planes (cross sections), resulting in a 3D geometric model of the object (Fig. 2(a)) and the grids (Fig. 2(b)). A grid generator

We define the centerlines and the radii of the wing and body based on digitized side and top view images (Fig. 2(a)), and accordingly extract the outline of each section approximately by an elliptic curve in which two axes of the elliptic curves are determined by the two 2D pixel images. For the wing a uniform thickness 
is taken but with elliptic smoothing at the leading and trailing edge as well as at the tip. As illustrated in Fig. 2(a) where a top-view fruit fly with a computer model superimposed on the right half of the reconstructed geometric model is realistic matching well the image. For each grid block, a 2D O-type structured grid around a circular cylinder is generated in advance for the preceding mapping process; and an O-O type grid topology is employed for the wing and body blocks, which can minimize the grid number, resulting in a large reduction in computing time. Moreover, to generate high-quality grids for the wing base, the wing tip, and the head-thorax-tail of the body, the wing and body grid blocks are further divided into three zones, e.g., the base zone, the wing zone and the tip zone for a wing block (Fig. 2(b)). Grids in the base-and tip-zone are then generated using Eriksson's Transfinite Interpolation method [10]. The grid is clustered to the wing-and body-surface with the minimum grid spacing adjacent to the wing surface controlled by a formula $\delta_{\text {min }}=0.1 c_{m} / \sqrt{R e}$, where $c_{m}$ is mean chord length of the wing and $R e$ the $c_{m}$-based Reynolds number. The wing block and the body block have an outside boundary of approximately two and twenty times of the mean chord length $c_{m}$, respectively.

\section{Multi-block and overset grid}

Insect flapping flight features multiple morphology of a two or four winged body, complex geometry and complicated movements of flapping wings and body. To deal with such complexity we use a multi-blocked, overset-grid method based on a chimera grid scheme [12]. The chimera scheme offers several advantages: 1) the use of body-fitted structured grids for each block and hence elimination of the burden of the grid generation of multiple, complex and moving objects; 2) the use of efficient block flow solver for the multi-blocked grid and associated boundary conditions; and 3) the use of interpolation for communication among overlapping grids which allows grids to be moved relative to each other [12]. For an insect-like model with flapping wings that move and/or deform with time, notably the wing-fitted structured grid must be regenerated in a timely manner, usually referred as 'grid assembly', and hence the communication among the moved and/or deformed grids needs to be re-established accordingly. To ensure the interpolation for communication among overlapping grids we hereby introduce a three-blocked, composite grid system (Fig. 
2, Fig. 3) for the two-winged insect model, which is composed of a body-fitted grid and two wing-fitted sub-grids which are completely embedded in the body-fitted grid.

\section{Grid assembly}

The grid assembly may be considered as pre-processing techniques for the flow solvers because the flow solver on each grid requires appropriate boundary conditions being supplied at the boundaries where the grid overlaps another grid. As shown in Fig. 3, whenever the grid around the wing overlaps another grid around the body or another wing, some grid points may lie inside the wing and/or body and thus will be outside of the flow field to be solved. These grid points are removed from the flow solutions on the grid by generating holes into any grids overlapping the surface of the wing or body. During holes generation, regions of the overlapping grids are marked as invalid, which creates additional boundaries within the grid system; and collectively, the grid points on the fringe of the holes and the grid points on the outer boundaries of the embedded grids are referred to as "inter-grid boundary points (IGBPs)". The boundary conditions required at the IGBPs, namely, the fortified boundary (Fig. 3), are supplied by interpolating the flow solution from any overlapping grids.

\section{Interpolation stencils}

In order to establish overlapped grid communications we need to represent point-volume geometric relationships to determine if a point lies inside a cell or grid as well as what is an appropriate interpolation stencil [12]. In this study, we divides the body-fitted grid into three zones including a head zone, a thorax zone and a tail zone, so as to classify the corresponding grid points (cells) relative to each zone (or block); and then, in each zone, the point-volume (cell) geometric relationship is established by determining the corresponding cell (volume) where the interpolation point lies.

Once an interpolation stencil is identified, the following tri-linear interpolation technique is used: for a curvilinear grid defined by the coordinates of the intersections of three families of boundary conforming grid lines denoted by $(\xi, \eta, \zeta)$, the coordinates at any point within a cell can be calculated such that: 


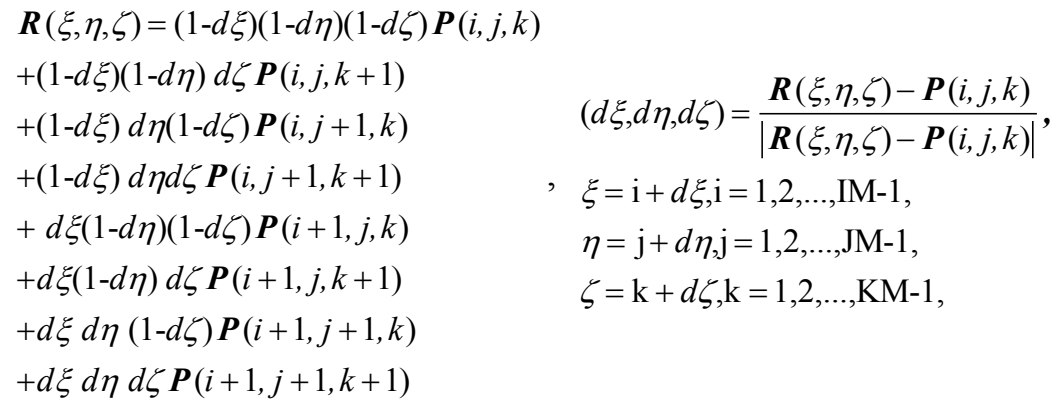

where $\boldsymbol{R}(\xi, \eta, \zeta)$ is unknown of a piecewise continuous function over the entire grid; $\boldsymbol{P}(i, j, k), \boldsymbol{P}(i, j, k+1)$, $\ldots$ are the known coordinates of the eight corners of a cell whereas the index $(i, j, k)$ denotes the 3D equivalent of the lower left corner of the cell; $(\delta \xi, \delta \eta, \delta \zeta)$ vary between 0 and 1 throughout the cell. For every known $\boldsymbol{P}$ that lies within a cell, there exists some $(\xi, \eta, \zeta)$ such that $\boldsymbol{P}=\boldsymbol{R}(\xi, \eta, \zeta)$, which is determined by using Newton's method to minimize the nonlinear function $\boldsymbol{F}=\boldsymbol{R}(\xi, \eta, \zeta)-\boldsymbol{P}$ iteratively, such that:

$$
\begin{array}{r}
\frac{\partial \boldsymbol{F}}{\partial \boldsymbol{\xi}}=\left(\mathrm{C}_{1}+d \boldsymbol{\eta} \mathrm{C}_{3}\right)+d \boldsymbol{\zeta}\left(\mathrm{C}_{5}+d \boldsymbol{\eta} \mathrm{C}_{7}\right) \\
\boldsymbol{\xi}^{m+1}=\boldsymbol{\xi}^{m}-\left[\frac{\partial \boldsymbol{F}^{m}}{\partial \boldsymbol{\xi}^{m}}\right]^{-1} \boldsymbol{F}^{m}, \frac{\partial \boldsymbol{F}}{\partial \boldsymbol{\eta}}=\left(\mathrm{C}_{2}+d \boldsymbol{\xi} \mathrm{C}_{3}\right)+d \boldsymbol{\zeta}\left(\mathrm{C}_{6}+d \boldsymbol{\xi} \mathrm{C}_{7}\right) \\
\frac{\partial \boldsymbol{F}}{\partial \boldsymbol{\zeta}}=\left(\mathrm{C}_{4}+d \boldsymbol{\xi} \mathrm{C}_{5}\right)+d \boldsymbol{\eta}\left(\mathrm{C}_{6}+d \boldsymbol{\xi} \mathrm{C}_{7}\right)
\end{array}
$$

where $\xi^{n}$ is the curvilinear vector $(\xi, \eta, \zeta), m$ is the Newton iteration counter, and the jacobian matrix is calculated using the following coefficients:

$$
\begin{aligned}
& C_{1}=\boldsymbol{P}(i+1, j, k)-\boldsymbol{P}(i, j, k), C_{2}=\boldsymbol{P}(i, j+1, k)-\boldsymbol{P}(i, j, k), \\
& C_{3}=\boldsymbol{P}(i+1, j+1, k)-\boldsymbol{P}(i, j+1, k)-C_{1}, C_{4}=\boldsymbol{P}(i, j, k+1)-\boldsymbol{P}(i, j, k), \\
& C_{5}=\boldsymbol{P}(i+1, j, k+1)-\boldsymbol{P}(i, j, k+1)-C_{1}, C_{6}=\boldsymbol{P}(i, j+1, k+1)-\boldsymbol{P}(i, j, k+1)-C_{2}, \\
& C_{7}=\boldsymbol{P}(i+1, j+1, k+1)-\boldsymbol{P}(i, j+1, k+1)-\boldsymbol{P}(i+1, j, k+1)+\boldsymbol{P}(i, j, k+1)-C_{3} .
\end{aligned}
$$

Newton's method needs a good starting point, which is chosen as $\xi^{0}=i, \eta^{0}=j, \zeta^{\theta}=k$ for each iteration. As the curvilinear coordinates $\xi$ are updated with Eq. (7), if $\delta \xi$ exceeds the range of 0 - 1 then search proceeds to a neighboring cell and the jacobian matrix, as well as the corners of the containing cell, must be updated, which is referred to as "stencil jump".

\section{Kinematic modeling}

Flapping motion 
Kinematics of insect flapping flight as illustrated in Fig. 1 consists of wingbeat and body kinematics. The insect body, if assumed to be rigid during flapping motion, is represented by the inclination of the body (or, body angle $\chi$ ) and the stroke plane angle $\beta$, which normally vary at different flight speeds. The wingbeat kinematics can then be described by three flapping angles within the stroke plane. Here, as shown in Fig. 4(a), 4(b), a general definition of the positional angle, the elevation angle and the AoA for a hovering fruitfly and a hovering hawkmoth, all in rad, are expressed using the Fourier series, such as:

$$
\begin{aligned}
& \phi(t)=\sum_{n=0}^{3}\left[\phi_{c n} \cos (n \omega t)+\phi_{s n} \sin (n \omega t)\right] \\
& \theta(t)=\sum_{n=0}^{3}\left[\theta_{c n} \cos (n \omega t)+\theta_{s n} \sin (n \omega t)\right], \\
& \alpha(t)=\sum_{n=0}^{3}\left[\alpha_{c n} \cos (n \omega t)+\alpha_{s n} \sin (n \omega t)\right]
\end{aligned}
$$

where $\omega$ is the flapping frequency; $n$ an integer varying from 0 to 3 ; and the coefficients $\phi_{c n, s n}, \theta_{c n, s n}, \alpha_{c n, s n}$ can be determined from the measured kinematic data [9-10, 31-32].

The kinematic modeling of the wing and body, if combined with the morphological modeling method with each cross section non-deformable, can be transferred to define a number sets of rotational axes at wing leading edge and body centerlines, which vary with time. Therefore, given $N(1,2,3, n, \ldots, N)$ sets of images of the wing and body in a complete beat cycle, one may extract the centerlines (or rotational axes for the wing) and the outline of each cross section through frame-by-frame image processing, and, by using the present method, integrates the morphological-and kinematic-modeling [20].

\section{Dynamic Regridding}

Since 3D motions of the flapping wing and the body, in general, result in large wing deformations and 6DOF (degree of freedom) body displacements, with a specific focus on robustness and efficiency of dynamic regridding, we developed a method by: 1) firstly rotating initial grids in the wing-fitted system according to a 'rigid' feathering motion; 2) secondly rotating the feathering-based grids according to a 'rigid' flapping motion; and 3) finally rotating the feathering-and flapping-based grids according to an elevation motion. Hence the regridding process is conducted by rigidly rotating all the initial grid points in the wing-fitted system $\left(x_{w}, y_{w}, z_{w}\right)$ (Fig. 1) with respect to a pivot point $\left(O_{w}\right)$ at the wing base, such that: 


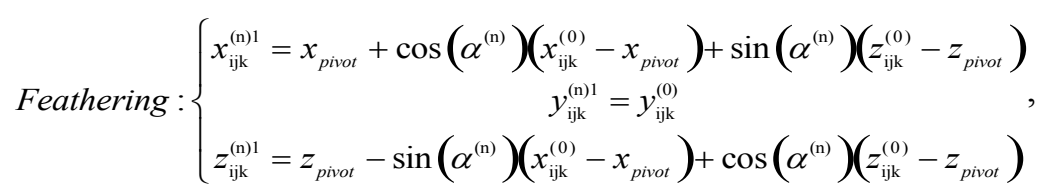

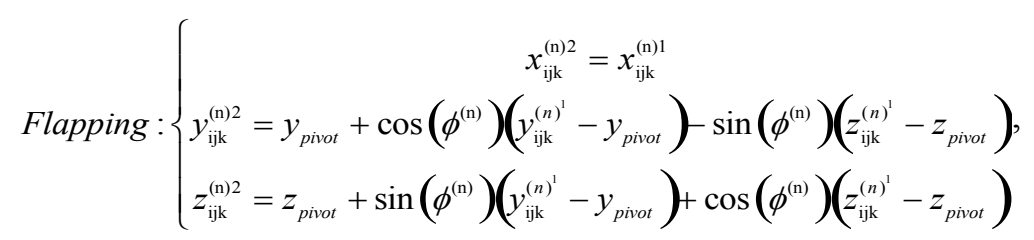

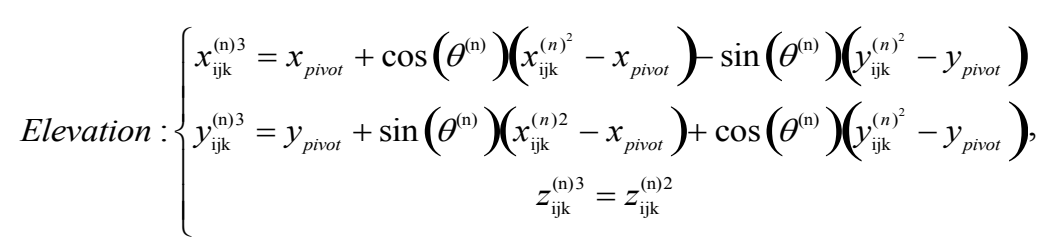

where superscript $n$ denotes the $n$-th time step; superscript 1,2, 3 represents the step due to the featheringflapping-elevation motions, respectively; $\operatorname{subscript}(i, j, k)$ expresses the grid point; and subscript pivot denotes the pivot point.

A flying body if treated as rigid is of 6DOF in a free flight. Thus the dynamic regridding for such a freely flying flyer may be implemented in two-fold: 1) transferring the initial grids by a displacement that the body moved; and 2) rotating grids in the whole body-fitted system $\left(x_{b}, y_{b}, z_{b}\right)$ according to the pitch-roll-yaw angles.

\section{Transformation among wing-fixed, body-fixed, and global coordinate systems}

Aiming at solving a general problem of insect free flight, we utilize three coordinate systems as depicted in Fig. 1: 1) the wing-fixed system which moves and deforms with the wing flapping movements; 2) the body-fixed system which moves as a rigid body in a 6DOF fashion; and 3) the global system which represents location and posture of the body. Three axes of the wing-fixed system are initially aligned with the body-fixed system. Since most insects flap their right and left wings in a symmetric way the wing-fixed coordinates is initially defined merely for the right wing.

Rotation of the wing-fixed system relative to the body-fixed system is defined by the AoA $(\alpha)$, the positional angle $(\phi)$, and the elevation angle $(\theta)$ (Fig. 1 and Fig. 4) while rotation of the body-fixed system relative to the global system is described by the three Euler angles of pitch $(\beta)$, roll $(\psi)$, and yaw $(\gamma)$. As described above, the three wing-fixed coordinate axes rotate in a step-by-step way by $\alpha$ about the $y_{b}$ axis, $\phi$ 
about the $x_{b}$ axis, and $\theta$ about the $z_{b}$ axis, which may be written in a matrix form, such that:

$$
\begin{gathered}
\text { Feathering : }\left[\begin{array}{c}
x_{b}^{\alpha} \\
y_{b}^{\alpha} \\
z_{b}^{\alpha}
\end{array}\right]=\left[\begin{array}{l}
x_{b}^{\text {pivot }} \\
y_{b}^{\text {pivot }} \\
z_{b}^{\text {pivot }}
\end{array}\right]+\left[\begin{array}{ccc}
\cos \alpha & 0 & \sin \alpha \\
0 & 1 & 0 \\
-\sin \alpha & 0 & \cos \alpha
\end{array}\right]\left(\left[\begin{array}{l}
x_{w} \\
y_{w} \\
z_{w}
\end{array}\right]-\left[\begin{array}{l}
x_{b}^{\text {pivot }} \\
y_{b}^{\text {pivot }} \\
z_{b}^{\text {pivot }}
\end{array}\right]\right), \mathrm{M}_{R y}(\alpha)=\left[\begin{array}{ccc}
\cos \alpha & 0 & \sin \alpha \\
0 & 1 & 0 \\
-\sin \alpha & 0 & \cos \alpha
\end{array}\right], \\
\text { Flapping: }\left[\begin{array}{l}
x_{b}^{\alpha \phi} \\
y_{b}^{\alpha \phi} \\
z_{b}^{\alpha \phi}
\end{array}\right]=\left[\begin{array}{l}
x_{b}^{\text {pivot }} \\
y_{b}^{\text {pivot }} \\
z_{b}^{\text {pivot }}
\end{array}\right]+\left[\begin{array}{ccc}
1 & 0 & 0 \\
0 & \cos \phi & -\sin \phi \\
0 & \sin \phi & \cos \phi
\end{array}\right]\left(\left[\begin{array}{l}
x_{b}^{\alpha} \\
y_{b}^{\alpha} \\
z_{b}^{\alpha}
\end{array}\right]-\left[\begin{array}{l}
x_{b}^{\text {pivot }} \\
y_{b}^{\text {pivot }} \\
z_{b}^{\text {pivot }}
\end{array}\right]\right), \mathrm{M}_{R x}(\phi)=\left[\begin{array}{ccc}
1 & 0 & 0 \\
0 & \cos \phi & -\sin \phi \\
0 & \sin \phi & \cos \phi
\end{array}\right], \\
\text { Elevation: }\left[\begin{array}{c}
x_{b}^{\alpha \phi \theta} \\
y_{b}^{\alpha \phi \theta} \\
z_{b}^{\alpha \phi \theta}
\end{array}\right]=\left[\begin{array}{l}
x_{b}^{\text {pivot }} \\
y_{b}^{\text {pivot }} \\
z_{b}^{\text {pivot }}
\end{array}\right]+\left[\begin{array}{ccc}
\cos \theta & -\sin \theta & 0 \\
\sin \theta & \cos \theta & 0 \\
0 & 0 & 1
\end{array}\right]\left(\left[\begin{array}{l}
x_{b}^{\alpha \phi} \\
y_{b}^{\alpha \phi} \\
z_{b}^{\alpha \phi}
\end{array}\right]-\left[\begin{array}{l}
x_{b}^{\text {pivot }} \\
y_{b}^{\text {pivot }} \\
z_{b}^{\text {pivot }}
\end{array}\right]\right), \mathrm{M}_{R z}(\theta)=\left[\begin{array}{ccc}
\cos \theta & -\sin \theta & 0 \\
\sin \theta & \cos \theta & 0 \\
0 & 0 & 1
\end{array}\right],
\end{gathered}
$$

where the superscripts $(\alpha, \phi, \theta)$ represent the transient coordinates during the feather-flap-elevation rotation.

With the notation $\boldsymbol{M}_{\boldsymbol{R} y}(\alpha), \boldsymbol{M}_{x y}(\phi), \boldsymbol{M}_{\boldsymbol{R} z}(\theta)$ representing rotational transformation matrices constructed for the rotations about the $\mathrm{y}$-, $\mathrm{x}$-and $\mathrm{z}$-axis, a complete transformation from the wing-fixed system to the body-fixed system can be written as:

$$
\left[\mathrm{M}_{R y}(\alpha) \mathrm{M}_{R x}(\phi) \mathrm{M}_{R z}(\theta)\right]\left(\left[\begin{array}{c}
x_{w} \\
y_{w} \\
z_{w}
\end{array}\right]-\left[\begin{array}{c}
x_{b}^{\text {pivot }} \\
y_{b}^{\text {pivot }} \\
z_{b}^{\text {pivot }}
\end{array}\right]\right)+\left[\begin{array}{c}
x_{b}^{\text {pivot }} \\
y_{b}^{\text {piot }} \\
z_{b}^{\text {piot }}
\end{array}\right]=\left[\begin{array}{c}
x_{b}^{\alpha \phi \theta} \\
y_{b}^{\alpha \phi \theta} \\
z_{b}^{\alpha \phi \theta}
\end{array}\right]
$$

where

$$
\mathrm{M}(\alpha, \phi, \theta)=\left[\mathrm{M}_{R y}(\alpha) \mathrm{M}_{R x}(\phi) \mathrm{M}_{R z}(\theta)\right]=\left[\begin{array}{ccc}
\cos \alpha \cos \theta-\sin \alpha \sin \theta \sin \phi & -\sin \theta \sin \phi & \sin \alpha \cos \theta+\cos \alpha \sin \theta \sin \phi \\
\cos \alpha \sin \theta+\sin \alpha \cos \theta \sin \phi & \cos \theta \cos \phi & \sin \alpha \sin \theta-\cos \alpha \cos \theta \sin \phi \\
-\sin \alpha \cos \phi & \sin \phi & \cos \alpha \cos \phi
\end{array}\right]
$$

Since the rotational transformations are orthonormal the inverse transform is equal to the transpose, and hence the complete transformation from the body-fixed system to the wing-fixed system can be written as:

$$
\left[\mathrm{M}_{R y}(\alpha)^{T} \mathrm{M}_{R x}(\phi)^{T} \mathrm{M}_{R z}(\theta)^{T}\right]\left(\left[\begin{array}{c}
x_{b}^{\alpha \phi \theta} \\
y_{b}^{\alpha \phi \theta} \\
z_{b}^{\alpha \phi \theta}
\end{array}\right]-\left[\begin{array}{c}
x_{b}^{\text {piot }} \\
y_{b}^{\text {piot }} \\
z_{b}^{\text {piot }}
\end{array}\right]\right)+\left[\begin{array}{l}
x_{b}^{\text {piot }} \\
y_{b}^{\text {piot }} \\
z_{b}^{\text {piot }}
\end{array}\right]=\left[\begin{array}{l}
x_{w} \\
y_{w} \\
z_{w}
\end{array}\right]
$$

where

$$
\mathrm{M}(\alpha, \phi, \theta)^{T}=\left[\begin{array}{ccc}
\cos \alpha \cos \theta & \cos \alpha \sin \theta+\sin \alpha \cos \theta \sin \phi & -\sin \alpha \cos \phi \\
-\sin \theta \cos \phi & \cos \theta \cos \phi & \sin \phi \\
\sin \alpha \cos \theta+\cos \alpha \sin \theta \sin \phi & \sin \alpha \sin \theta-\cos \alpha \cos \theta \sin \phi & \cos \alpha \cos \phi
\end{array}\right]
$$


Similarly, the complete transformation matrix due to the rotation of the body-fixed system $x_{b} y_{b} z_{b}$ relative to the global system $X Y Z$ can be written as:

$$
\left[\mathrm{M}_{R y}(\beta) \mathrm{M}_{R x}(\psi) \mathrm{M}_{R z}(\gamma)\right]\left(\left[\begin{array}{c}
x_{b} \\
y_{b} \\
z_{b}
\end{array}\right]-\left[\begin{array}{c}
X^{O b} \\
Y^{o b} \\
Z^{o b}
\end{array}\right]\right)+\left[\begin{array}{c}
X^{o b} \\
Y^{o b} \\
Z^{o b}
\end{array}\right]=\left[\begin{array}{c}
X^{\beta \omega y} \\
Y^{\beta \psi \gamma} \\
Z^{\beta \psi \gamma}
\end{array}\right]
$$

where

$$
\mathrm{M}(\beta, \psi, \gamma)=\left[\mathrm{M}_{R y}(\beta) \mathrm{M}_{R x}(\psi) \mathrm{M}_{R z}(\gamma)\right]=\left[\begin{array}{ccc}
\cos \beta \cos \gamma-\sin \beta \sin \theta \sin \psi & -\sin \theta \sin \psi & \sin \beta \cos \gamma+\cos \beta \sin \gamma \sin \psi \\
\cos \beta \sin \gamma+\sin \beta \cos \theta \sin \psi & \cos \theta \cos \psi & \sin \beta \sin \gamma-\cos \beta \cos \gamma \sin \psi \\
-\sin \beta \cos \psi & \sin \psi & \cos \beta \cos \psi
\end{array}\right]
$$

Note that the body is assumed to rotate about the origin $O_{b}$, and the transpose of the matrix, i.e., the complete transformation from the global system to the body-fixed system can be expressed as:

$$
\left[\mathrm{M}_{R y}(\beta)^{T} \mathrm{M}_{R x}(\psi)^{T} \mathrm{M}_{R z}(\gamma)^{T}\right]\left(\left[\begin{array}{c}
X^{\beta \psi \gamma} \\
Y^{\beta y \gamma} \\
Z^{\beta \psi \gamma}
\end{array}\right]-\left[\begin{array}{c}
X^{o b} \\
Y^{O b} \\
Z^{o b}
\end{array}\right]\right)+\left[\begin{array}{c}
X^{O b} \\
Y^{o b} \\
Z^{O b}
\end{array}\right]=\left[\begin{array}{c}
x_{b} \\
y_{b} \\
z_{b}
\end{array}\right]
$$

where

$$
\mathrm{M}(\beta, \psi, \gamma)^{T}=\left[\begin{array}{ccc}
\cos \beta \cos \gamma & \cos \beta \sin \gamma+\sin \beta \cos \gamma \sin \psi & -\sin \beta \cos \psi \\
-\sin \gamma \cos \psi & \cos \gamma \cos \psi & \sin \psi \\
\sin \beta \cos \gamma+\cos \beta \sin \gamma \sin \psi & \sin \beta \sin \gamma-\cos \beta \cos \gamma \sin \psi & \cos \beta \cos \psi
\end{array}\right]
$$

\section{Stroke plane and body angle}

As shown in Fig. 1, Fig. 4, we need to define an initial relationship between the wing-fixed and body-fixed systems during the coordinate transformation by taking into account the stroke plane angle $\eta$ as the inclination angle of the $y_{w} z_{w}$ plane relative to horizontal. Hence the stroke plane-based coordinate transformations among the three coordinate systems, can be conducted, such that:

$$
\left[\mathrm{M}_{R y}(\eta)\right]\left(\left[\begin{array}{c}
x_{b}^{\alpha \phi \theta} \\
y_{b}^{\alpha \phi \theta} \\
z_{b}^{\alpha \phi \theta}
\end{array}\right]-\left[\begin{array}{c}
X^{o b} \\
Y^{o b} \\
Z^{o b}
\end{array}\right]\right)+\left[\begin{array}{c}
X^{o b} \\
Y^{o b} \\
Z^{o b}
\end{array}\right]=\left[\begin{array}{c}
X^{\eta} \\
Y^{\eta} \\
Z^{\eta}
\end{array}\right], \mathrm{M}_{R y}(\eta)=\left[\begin{array}{ccc}
\cos \left(\frac{\pi}{2}-\eta\right) & 0 & \sin \left(\frac{\pi}{2}-\eta\right) \\
0 & 1 & 0 \\
-\sin \left(\frac{\pi}{2}-\eta\right) & 0 & \cos \left(\frac{\pi}{2}-\eta\right)
\end{array}\right] .
$$

Furthermore, the coordinate transformation between the body-fixed and global coordinate systems, by defining an initial relative inclination angle of the body, $\chi$, can be written as: 


$$
\left[\mathrm{M}_{R y}(\chi)\right]\left(\left[\begin{array}{l}
x_{b} \\
y_{b} \\
z_{b}
\end{array}\right]-\left[\begin{array}{l}
X^{O b} \\
Y^{O b} \\
Z^{O b}
\end{array}\right]\right)+\left[\begin{array}{c}
X^{O b} \\
Y^{O b} \\
Z^{O b}
\end{array}\right]=\left[\begin{array}{l}
X^{\chi} \\
Y^{\chi} \\
Z^{\chi}
\end{array}\right], \mathrm{M}_{R y}(\eta)=\left[\begin{array}{ccc}
\cos \chi & 0 & \sin \chi \\
0 & 1 & 0 \\
-\sin \chi & 0 & \cos \chi
\end{array}\right] .
$$

Note that the coordinate transformations or the rotations due to the stroke plane angle $\eta$ and the body angle $\chi$ are, in general, independent of the pitch $(\beta)$, roll $(\psi)$, and yaw $(\gamma)$ angles, and hence they must be added as an apparent pitch angle of $\beta+(\pi / 2-\eta)$ for the wing and $\beta+\chi$ for the body, respectively.

\section{E. Scaling in flapping flight}

Scaling in insect flapping flight is a useful measure to predict the effects of viscous-and inertial-aerodynamic force and unsteadiness on aerodynamic features. Three main dimensionless parameters in flapping-flight scaling are: 1) the Reynolds number $(R e)$, which represents a ratio of inertial and viscous force, 2) the Strouhal number (St) for forward flight which describes the relative influence of forward versus flapping speeds, and 3) the reduced frequency $(k)$, which describes the rotational versus translational speeds during flapping movement. Together with geometric and kinematic similarities, the three parameters $R e, S t$ and $k$ are sufficient to define the aerodynamic similarity for a rigid wing.

\section{Reynolds number}

Given a reference length $L_{r e f}$ and a reference velocity $U_{r e f}$, the Reynolds number may be defined as

$$
\operatorname{Re}=\frac{U_{r e f} L_{r e f}}{v}
$$

where $v$ is the kinematic viscosity. The mean chord length $c_{m}$ is used as the reference length $L_{r e f}$; the reference velocity $U_{\text {ref }}$ is defined by a forward velocity $U$ in forward flight but a mean wing tip velocity in hovering flight, which is proportional to $U_{\text {ref }}=\omega R$, where $R$ is the wing length and $\omega$ is the mean angular velocity of the wing ( $\omega=2 \Phi f$, where $\Phi$ is the wing beat amplitude and $f$ is the flapping frequency). Therefore, the Reynolds number in hovering flight can be reformed as

$$
R e=\frac{U_{r e f} L_{r e f}}{v}=\frac{2 \Phi f R c_{m}}{v}=\frac{\Phi f R^{2}}{v}\left(\frac{4}{A R}\right)
$$

where the aspect ratio $A R$ is in a form of $A R=(2 R)^{2} / S$, with a wing area of $S=2 R c_{m}$. Note that the Reynolds 
number here is proportional to the wing beat amplitude, $\Phi$, the flapping frequency, $f$, a square of the wing length, $R^{2}$, but proportional inversely to the aspect ratio of the wing, $A R$. In insect flights, the wing beat amplitude and the aspect ratio of the wing do not vary significantly, but the flapping frequency increases and the wing length decreases rapidly when the insect size is reduced, which, in general, results in a wide $R e$ range from $O\left(10^{1}\right)$ for tiny thrips, $O\left(10^{2}\right)$ for fruitflies, and up to $O\left(10^{4}\right)$ for moths. In addition, given a geometrically similar wing model that undergoes flapping hovering with the same wing beat amplitude, the same product of $f R^{2}$ can preserve the same Reynolds number. This implies that a scaled-up but low flapping frequency wing model may be built mechanically to mimic insect flapping flight based on the aerodynamic similarity of the rigid wing $[8,11]$.

We here give three typical Reynolds numbers in insect hovering flights. For a hawkmoth [7-10], $c_{m}=1.83$ $\mathrm{cm}, R=4.83 \mathrm{~cm}, \Phi=2.0 \mathrm{rad}, f=26.1 \mathrm{~s}^{-1}, v=1.5 \times 10^{-5} \mathrm{~m}^{2} / \mathrm{s}$ (kinematic viscosity of air), so $R e$ is calculated to be 6300 ; for a honeybee [21], $c_{m}=2.39 \mathrm{~mm}, R=9.7 \mathrm{~mm}, \Phi=1.58 \mathrm{rad}, f=230 \mathrm{~s}^{-1}$, so $R e$ is 1134 ; and for a fruitfly [16], $c_{m}=0.78 \mathrm{~mm}, R=2.39 \mathrm{~mm}, \Phi=2.44 \mathrm{rad}, f=218 \mathrm{~s}^{-1}$, so $R e$ is 134 .

\section{Strouhal number and reduced frequency}

In flapping flight, the Strouhal number is well known for characterizing the vortex dynamics and shedding behavior and normally defined as:

$$
S t=\frac{f L_{r e f}}{U_{r e f}}=\frac{f A}{U},
$$

where $f$ is the flapping frequency, $A$ is the flapping amplitude, and $U$ is the forward velocity. This definition describes a ratio between the oscillating (flapping) speed $(f A)$ and the forward speed $(U)$, which is normally used as a measure for propulsive efficiency in flying animals.

The reduced frequency that normally characterizing rotational versus translational speeds, is defined as:

$$
k=\frac{2 \pi f L_{r e f}}{2 U_{r e f}}=\frac{\pi f c_{m}}{U}
$$

In hovering flights, using the mean wing tip velocity $\left(U_{r e f}=2 \Phi f R\right)$ as the reference velocity, the reduced frequency can be reformed as 


$$
k=\frac{\pi f c_{m}}{U}=\frac{\pi c_{m}}{2 \Phi R}=\frac{\pi}{\Phi A R}
$$

Note that the reduced frequency $k$ is proportional inversely to the beat amplitude $\Phi$ and the aspect ratio $A R$ of the wing. Therefore, for a geometrically similar wing model in hovering, if the wing beat amplitude and the product of $f R^{2}$ can preserve simultaneously, $R e$ and $k$ can preserve and hence the aerodynamic similarity can be established accordingly.

Using the reduced frequency the wing kinematics, the Eq. (9) can be rewritten as:

$$
\begin{aligned}
& \phi(t)=\sum_{n=0}^{3}\left[\phi_{c n} \cos (2 k n t)+\phi_{s n} \sin (2 k n t)\right] \\
& \theta(t)=\sum_{n=0}^{3}\left[\theta_{c n} \cos (2 k n t)+\theta_{s n} \sin (2 k n t)\right] \\
& \alpha(t)=\sum_{n=0}^{3}\left[\alpha_{c n} \cos (2 k n t)+\alpha_{s n} \sin (2 k n t)\right]
\end{aligned}
$$

where $t$ is a dimensionless time, which is non-dimensionalized by a reference time of $T_{r e f}=L_{r e f} / U_{\text {ref }}$, resulting in a dimensionless period of $\pi / k$. Note that, if the period, $1 / f$, is used for such non-dimensionalization, the $2 k n t$ will be replaced by $2 \pi t / n$ in the preceding equations with a dimensionless period of 1.0.

We here further give reduced frequencies for the three typical insects in hovering as described above. For the hawkmoth, $k$ is calculated to be 0.3 with a period of 10.5 ; for the honeybee, $k$ is 0.244 with a period of 12.9; and for the fruitfly, $k$ is 0.21 with a period of 15.0 . [7-10, 16, 21]

\section{F. Multi-blocked, fortified solutions to the Navier-Stokes equations}

A general formulation of the multi-blocked, overset grid, fortified solutions to the NS equations is performed in the global system (XYZ) as depicted in Fig. 1(a). The algorithm employed here is based on a single NS solver as can be found in $[10,20]$ but is extended to a multi-blocked, overset-grid system by means of the fortified solution algorithm [5].

\section{Governing equations}

The governing equations are the three-dimensional, incompressible, unsteady NS equations written in strong conservation form for mass and momentum. The artificial compressibility method is used by adding a pseudo time derivative of pressure to the equation of continuity. For an arbitrary deformable control volume 
$V(t)$, the nondimensionalized governing equations are

$$
\int_{V_{(\theta)}}\left(\frac{\partial \boldsymbol{Q}}{\partial t}+\frac{\partial \boldsymbol{q}}{\partial \tau}\right) d V+\int_{V(\theta)}\left(\frac{\check{Z} \boldsymbol{F}}{\check{Z} x}+\frac{\check{Z} \boldsymbol{G}}{\check{Z} y}+\frac{\check{Z} \boldsymbol{H}}{\check{Z} z}+\frac{\check{Z} \boldsymbol{F}_{v}}{\check{Z} x}+\frac{\check{Z} \boldsymbol{G}_{v}}{\check{Z} y}+\frac{\check{Z} \boldsymbol{H}_{v}}{\check{Z} z}\right) d V=0
$$

where

$$
\boldsymbol{Q}=\left[\begin{array}{c}
u \\
v \\
w \\
0
\end{array}\right] \boldsymbol{q}=\left[\begin{array}{c}
u \\
v \\
w \\
p
\end{array}\right], \boldsymbol{F}=\left[\begin{array}{c}
u^{2}+p \\
u v \\
u w \\
\lambda u
\end{array}\right] \boldsymbol{G}=\left[\begin{array}{c}
v u \\
v^{2}+p \\
v w \\
\lambda v
\end{array}\right] \boldsymbol{H}=\left[\begin{array}{c}
w u \\
w v \\
w^{2}+p \\
\lambda w
\end{array}\right], \boldsymbol{F}_{v}=-\frac{1}{R e}\left[\begin{array}{c}
2 u_{x} \\
u_{y}+v_{x} \\
u_{z}+w_{x} \\
0
\end{array}\right] \boldsymbol{G}_{v}=-\frac{1}{R e}\left[\begin{array}{c}
v_{x}+u_{y} \\
2 v_{y} \\
v_{z}+w_{y} \\
0
\end{array}\right] \boldsymbol{H}_{v}=-\frac{1}{\operatorname{Re}}\left[\begin{array}{c}
w_{x}+u_{z} \\
w_{y}+v_{z} \\
2 w_{z} \\
0
\end{array}\right] .
$$

In the preceding equations, $\lambda$ is the pseudo-compressibility coefficient; $p$ is pressure; $u, v$, and $w$ are velocity components in Cartesian coordinate system $X, Y$, and $Z$; $t$ denotes physical time while $\tau$ is pseudo time; and $R e$ is the Reynolds number. Note that the term $\boldsymbol{q}$ associated with the pseudo time is designed for an inner-iteration at each physical time step, and will vanish when the divergence of velocity is driven to zero so as to satisfy the equation of continuity.

By introducing the generalized Reynolds transport theorem and by employing the Gauss integration theorem, an integrated form of the Eq. (27) in general curvilinear coordinate system is gained as

$$
\int_{V(t)} \frac{\partial \boldsymbol{q}}{\partial \tau} d V+\frac{\partial}{\partial t} \int_{V(t)} \boldsymbol{Q} d V+\prod_{S(t)}\left(\boldsymbol{f}-\boldsymbol{Q} \boldsymbol{u}_{g}\right) \cdot \boldsymbol{n} d S=0
$$

where $\boldsymbol{f}=\left(\boldsymbol{F}+\boldsymbol{F}_{\boldsymbol{v}}, \boldsymbol{G}+\boldsymbol{G}_{\boldsymbol{v}}, \boldsymbol{H}+\boldsymbol{H}_{v}\right) ; S(t)$ denotes the surface of the control volume; $\boldsymbol{n}=\left(n_{x}, n_{y}, n_{z}\right)$ are components of the unit outward normal vector corresponding to all the faces of the polyhedron cell; $\boldsymbol{u}_{g}$ is the local velocity of the moving cell surface. For a structured, boundary-fitted, and cell-centered storage architecture, we can further reform Eq. (28) in terms of the semi-discrete form, where $(i, j, k)$ denote the cell index, such that

$$
\frac{\partial}{\partial t}[V \boldsymbol{Q}]_{j j k}+\boldsymbol{R}_{i j k}+V_{i j k}\left(\frac{\partial \boldsymbol{q}}{\partial \tau}\right)_{i j k}=0
$$

where

$$
\begin{aligned}
& \boldsymbol{R}_{i j k}=\left(\hat{\boldsymbol{F}}+\hat{\boldsymbol{F}}_{v}\right)_{i+\frac{1}{2}, j, k}-\left(\hat{\boldsymbol{F}}+\hat{\boldsymbol{F}}_{v}\right)_{i-\frac{1}{2}, j, k} \quad \text { e.g. } \hat{\boldsymbol{F}}+\hat{\boldsymbol{F}}_{v}=\left(\boldsymbol{f}-\boldsymbol{Q} \boldsymbol{u}_{\boldsymbol{g}}\right) \cdot \boldsymbol{S}_{n}^{\xi} \\
& +\left(\hat{\boldsymbol{G}}+\hat{\boldsymbol{G}}_{v}\right)_{i, j+\frac{1}{2}, k}-\left(\hat{\boldsymbol{G}}+\hat{\boldsymbol{G}}_{v}\right)_{i, j-\frac{1}{2}, k} \text { and } \boldsymbol{S}_{n}^{x}=\left[S_{n x}^{\xi}, S_{n y}^{\xi}, S_{n z}^{\xi}\right], \boldsymbol{n}=\left[S_{n x}^{\xi}, S_{n y}^{\xi}, S_{n z}^{\xi}\right] / S \text {. } \\
& +\left(\hat{\boldsymbol{H}}+\hat{\boldsymbol{H}}_{v}\right)_{i, k, k+\frac{1}{2}}-\left(\hat{\boldsymbol{H}}+\hat{\boldsymbol{H}}_{v}\right)_{i, j, k \frac{1}{2}} \quad \boldsymbol{S}=\sqrt{S_{n x}^{\xi 2}+S_{n y}^{\xi 2}+S_{n z}^{\xi 2}} .
\end{aligned}
$$


The term $V_{i j k}$ is the volume of the cell $(i, j, k)$. Note that the unit outward normal vector $\boldsymbol{n}$ can be calculated using the areas of the cell faces, e.g., $S_{n}^{\xi}$ in $\xi$-direction. A detailed description of evaluation of the inviscid flux and the viscous flux can be found in $[10,20]$.

\section{Fortified solution algorithm}

The fortified solution algorithm based on the fortified NS approach [5] is achieved by adding forcing terms to the NS equations, for the semi-discrete form, such as:

$$
\frac{\partial}{\partial t}[V \boldsymbol{Q}]_{i j k}+\boldsymbol{R}_{i j k}+V_{i j k}\left(\frac{\partial \boldsymbol{q}}{\partial \tau}\right)_{i j k}=\sigma\left(\boldsymbol{q}_{f}-\boldsymbol{q}\right),
$$

where the switching parameter $\sigma$ is set to be sufficiently large, compared to all the other terms in the region, and where the solution $\boldsymbol{q}_{f}$ is available by the subset equations, and zero outside the region. For $\sigma>>1$, the added source term simply forces $\boldsymbol{q}=\boldsymbol{q}_{\boldsymbol{f}}$, otherwise it blends $\boldsymbol{q}$ with $\boldsymbol{q}_{\boldsymbol{f}}$. When $\sigma=0$, the equations go back to the ordinary NS equations. In a wider sense, this method provides a potential payoff to an improvement of both the efficiency and accuracy of any governing equations but not limited to the NS equations.

The Pade scheme is employed for the time integration

$$
\frac{\partial}{\partial t}=\frac{1}{\Delta t} \frac{\Delta}{1+\vartheta \Delta} \longrightarrow \frac{\Delta(V \boldsymbol{Q})_{i j k}^{(n+1)}-\Delta(V \boldsymbol{Q})_{i j k}^{(n)}}{\Delta t}=-\left\{\boldsymbol{R}_{i j k}+V_{i j k}\left(\frac{\partial \boldsymbol{q}}{\partial \tau}\right)_{i j k}-\sigma\left(\boldsymbol{q}_{f}-\boldsymbol{q}\right)\right\}^{(n+1)}
$$

where parameter $\vartheta$ is taken to be 0.5 for the implicit Euler scheme with second-order accuracy in time; $\Delta t$ is the time increment; and $\Delta \boldsymbol{q}=\boldsymbol{q}^{(n+1)}-\boldsymbol{q}^{(n)}$. Thus, the Eq. (30) can be discretized by replacing the time-related term with the Eq. (31), such that

$$
\Delta(V \boldsymbol{Q})_{i j k}^{(n)}+\vartheta \Delta t \Delta\left[\boldsymbol{R}_{j k}+V_{i j k}\left(\frac{\partial \boldsymbol{q}}{\partial \tau}\right)_{i j k}-\sigma\left(\boldsymbol{q}_{f}-\boldsymbol{q}\right)\right]^{(n)}=-\Delta t\left[\boldsymbol{R}_{i j k}+V_{i j k}\left(\frac{\partial \boldsymbol{q}}{\partial \tau}\right)_{i j k}-\sigma\left(\boldsymbol{q}_{f}-\boldsymbol{q}\right)\right]^{(m)}
$$

Since the volume of the cell may change with time due to the moving grid system, we assess the first term in Eq. (32) as

$$
\Delta(V \boldsymbol{Q})_{i j k}^{(n)}=(V \boldsymbol{Q})_{i j k}^{(n+1)}-(V \boldsymbol{Q})_{i j k}^{(n)}=V_{i j k}^{(n+1)} \Delta \boldsymbol{Q}_{i j k}^{(n)}+\Delta V_{i j k}^{(n)} \boldsymbol{Q}_{i j k}^{(n)} \approx V_{i j k}^{(n)} \Delta \boldsymbol{Q}_{i j k}^{(n)}+\Delta V_{i j k}^{(n)} \boldsymbol{Q}_{i j k}^{(n)}
$$

A problem rises here as to how to satisfy the so-called "Geometric Conservation Law", i.e., the conservation of momentum taken into Newton laws at each time step in term of evaluation of the increment of the volume 
$\Delta V_{i j k}(n)$ for the moving grid system. Considering the conservation of flux across the cell faces in an extreme case of solving uniform flow with the moving grid system, $\Delta V_{i j k}(n)$ can be explicitly expressed as

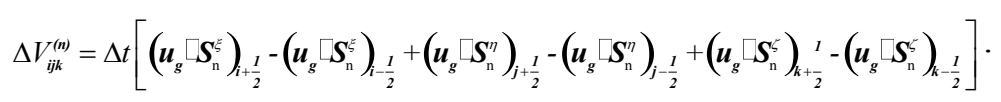

The pseudo time-related terms designed for the inner-iteration can be approximated as

$$
\vartheta \Delta t \Delta\left(V_{i j k} \frac{\partial \boldsymbol{q}}{\partial \tau}\right)^{(m)}+\Delta t\left(V_{i j k} \frac{\partial \boldsymbol{q}}{\partial \tau}\right)^{(m)}=\Delta t\left[(1-\vartheta)\left(V_{i j k} \frac{\partial \boldsymbol{q}}{\partial \tau}\right)^{(n)}+\vartheta V_{i j k}^{(n)} \frac{\partial \boldsymbol{q}^{(n+1)}}{\partial \tau}\right] \approx \Delta t V_{i j k}^{(n)} \frac{\partial \boldsymbol{q}^{(m)}}{\partial \tau}
$$

Note that the approximation $\left(\partial \boldsymbol{q}^{(\mathrm{n})} / \partial \tau=\partial \boldsymbol{q}^{(\mathrm{n}+1)} / \partial \tau\right)$ is reasonable because the pseudo time $\tau$ is for the inner-iteration and thus is dependent at each physical time step. Hence, the governing equations become

$$
\frac{\Delta \boldsymbol{Q}_{i j k}^{(n)}}{\Delta t}+\frac{\vartheta \Delta \boldsymbol{R}_{i j k}^{(n)}}{V_{j k k}^{(n)}(1+\vartheta \Delta t \sigma)}+\frac{1}{(1+\vartheta \Delta t \sigma)} \frac{\partial \boldsymbol{q}^{(n)}}{\partial \tau}=-\frac{\boldsymbol{R}_{i j k}^{(n)}}{V_{j i k}^{(n)}(1+\vartheta \Delta t \sigma)}-\frac{\Delta V_{i j k}^{(n)}}{\Delta t(1+\vartheta \Delta t \sigma) V_{i j k}^{(n)}} \boldsymbol{Q}_{i j k}^{(n)}+\frac{\sigma}{(1+\vartheta \Delta t \sigma)}\left(\boldsymbol{q}_{f}-\boldsymbol{q}\right) .
$$

The implicit Euler scheme is also employed for the pseudo time integration. With the differencing operator for the pseudo time, the governing equations can be reformed as

$$
\begin{aligned}
& \left\{\frac{\boldsymbol{I}}{\Delta \tau}+\frac{\vartheta}{(1+\vartheta \Delta t \sigma)}\left[\frac{\boldsymbol{I}_{a}}{\Delta t}\left(1+\frac{\Delta V_{j k}^{(j)}}{V_{j k}^{(i)}}\right)+\frac{1}{V_{i j k}^{((j)}} \frac{\partial \boldsymbol{R}_{j k}^{(m, m)}}{\partial \boldsymbol{q}}\right]\right\} \Delta \boldsymbol{q}_{j k}^{(m, m)}
\end{aligned}
$$

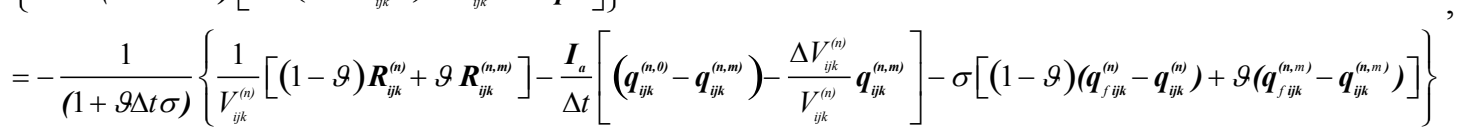

where $\Delta \tau$ is the pseudotime-step size and $\boldsymbol{I}_{\boldsymbol{a}}=[1,1,1,0]^{\mathrm{T}}$. Note that the forcing term is easily treated implicitly. In the region as an overlapping one between two grids where $\sigma$ is sufficiently large, the NS equations algorithm is turned off and reduces simply to $\boldsymbol{q}^{(\boldsymbol{n}+1)}=\boldsymbol{q}_{f}$, so the solution is fortified there. In the computational domain where $\sigma$ is set to zero, we have the algorithm reduced to the standard one. Note that the parameter is added to the diagonal term implicitly, which, if a positive larger $\sigma$ is taken, obviously is capable to enhance the stability of the algorithm.

In order to benefit from both lower memory and computational requirements for the solution of the Eq. (36), the approximated factorization method [10, 20], is used for the lift hand side (LHS), and hence, the Eq. (36) is rewritten as: 


$$
\begin{aligned}
& \left\{\boldsymbol{I}+\frac{\vartheta \Delta t \boldsymbol{I}_{t}}{(1+\vartheta \Delta t \sigma) V_{i j k}^{(n)}} \frac{\partial \boldsymbol{R}_{i j k}^{(n, m)}}{\partial \boldsymbol{q}}\right\}^{(\xi)}\left\{\boldsymbol{I}+\frac{\vartheta \Delta t \boldsymbol{I}_{t}}{(1+\vartheta \Delta t \sigma) \boldsymbol{V}_{i j k}^{(n)}} \frac{\partial \boldsymbol{R}_{i j k}^{(n, m)}}{\partial \boldsymbol{q}}\right\}^{(n)}\left\{\boldsymbol{I}+\frac{\vartheta \Delta t \boldsymbol{I}_{t}}{(1+\vartheta \Delta t \sigma) V_{i j k}^{(n)}} \frac{\partial \boldsymbol{R}_{i j k}^{(n, m)}}{\partial \boldsymbol{q}}\right\}^{(\zeta)} \Delta \Delta \boldsymbol{q}_{i j k}^{(n, m)}=
\end{aligned}
$$

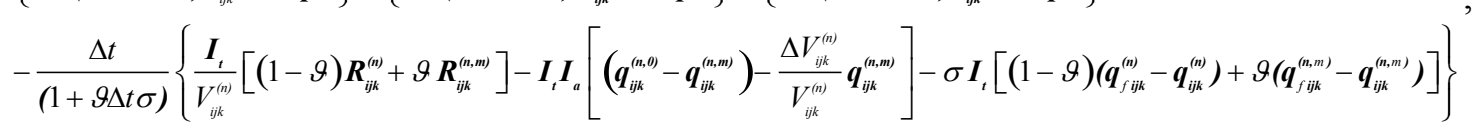

where $\boldsymbol{I}_{t}=(1 /(\vartheta+\Delta t / \Delta \tau), 1 /(\vartheta+\Delta t / \Delta \tau), 1 /(\vartheta+\Delta t / \Delta \tau), \Delta \tau / \Delta t)$, a diagonal matrix. The preceding equations can be further decomposed into three sweeps in the $\xi$-, $\eta$-, and $\zeta$-directions in computational domain. A linear system of equations is finally yielded, in which the discrete form of the matrix from the LHS is tridiagonally banded. (More details can be found in [10, 20])

\section{Boundary conditions}

The solutions to the multi-blocked, overset grid NS equations require specific boundary conditions at the overlapping zone stencils among grids, at the solid walls of dynamic flapping wings and body as well as at the far field outside boundary (Fig. 3).

For three single grid blocks in a two-winged insect model we solve the fortified NS equations (Eq. (30)) three times at each time step. For the holes inside the grid and the outermost two grid points, the $\boldsymbol{q}_{f}$ are specified there by $\boldsymbol{q}$ of overlapping zones with other single grids (another wing or body) at previous time step. Inside the computational domain except the holes and the single grid boundary $\boldsymbol{q}$ are equal to $\boldsymbol{q}_{\boldsymbol{f}}$ by setting $\sigma=0$.

On the body surface, the no-slip condition is used for the velocity components. To incorporate the dynamic effect due to the acceleration of the oscillating body (moving and/or deforming body surface), pressure divergence at the surface stencils is derived from the local momentum equation, such that

$$
(u, v, w)=\left(u_{\text {body }}, v_{\text {body }}, w_{\text {body }}\right), \frac{\partial p}{\partial \boldsymbol{n}}=-\boldsymbol{a}_{o} \llbracket \boldsymbol{n},
$$

where the velocity $\left(u_{b o d y}, v_{b o d y}, w_{b o d y}\right)$ and the acceleration $\left(\boldsymbol{a}_{\boldsymbol{o}}\right)$ on the solid wall are evaluated and updated using the renewed grids on the body surface at each time step.

For the background grid of the insect body we need to define appropriate boundary conditions at far field outside boundary. Consider that, when an insect hovers or flies forward at a speed $\boldsymbol{V}_{f}$, the boundary conditions for the velocity and the pressure may be given such as: 1) at upstream $\boldsymbol{V}(u, v, w)=\boldsymbol{V}_{\boldsymbol{f}}$ while pressure $p$ is set to zero; 2) at downstream zero-gradient condition is taken for both velocity and pressure, 
i.e., $\partial(u, v, w, p) / \partial \boldsymbol{n}=0$, where $\boldsymbol{n}$ is the unit outward normal vector at the outside boundary.

\section{G. Evaluation of flapping energetics: force, torque and power}

When the NS equations are solved for each block the aerodynamic forces $\boldsymbol{F}_{\text {aero }}\left(F_{x}, F_{y}, F_{z}\right)$ exerted on the wing and on the body are evaluated by a sum of inviscid and viscous flux over the wing surface or the body surface as

$$
\boldsymbol{F}_{\text {aero }}\left(F_{x}, F_{y}, F_{z}\right)=-\sum_{i}^{N}\left(\boldsymbol{F l u x} \boldsymbol{x}_{\text {invis }}+\boldsymbol{F l u x _ { \text { vis } }}\right)
$$

where $N$ denotes the cell number on the surface of the wing or the body. Note that, rather than directly integrating the velocity gradient-based stresses and the pressures over the wing or body surface this method can minimize the numerical error during the integration [10]. Furthermore, a sum of three sets of these aerodynamic forces for the two wings and the body results in three force components in the global inertial system, yielding vertical, horizontal and sideslip forces. In hovering flight we also call the vertical force and the horizontal force as the lift force and the drag force, respectively, which may be non-dimensionalized as

$$
\boldsymbol{F}_{\text {aero }}^{*}=\frac{\boldsymbol{F}_{\text {aero }}}{0.5 \rho U_{\text {ref }}^{2} S_{w}} \rightarrow C_{L}=\frac{\text { Lift }}{0.5 \rho U_{\text {ref }}^{2} S_{w}}, C_{D}=\frac{\text { Drag }}{0.5 \rho U_{r e f}^{2} S_{w}}
$$

where $U_{r e f}$ is the reference velocity, $\rho$ is the air density and $S_{w}$ is the planform area of a single wing.

Given the wing kinematics, according to Newton's second law, the non-dimensional inertial forces $\left(\boldsymbol{F}^{*}{ }_{\text {iner }}\right)$ acting upon the wing may be obtained as

$$
\boldsymbol{F}_{\text {iner }}=-\sum_{i} \frac{d\left(\rho_{\mathrm{w}} \Delta v_{i} \boldsymbol{v}_{i}^{*}(t)\right)}{d t} \rightarrow \boldsymbol{F}_{\text {iner }}^{*}=\frac{\boldsymbol{F}_{\text {iner }}}{0.5 \rho U_{r e f}^{2} S_{w}},
$$

where the wing mass density $\rho_{w}$ is assumed to be constant, $\Delta v_{i}$ is the volume of the cell constructed by eight grid points on the wing, and $\boldsymbol{v}_{i}{ }_{i}(t)$ is the computed wing velocities at the $i$-th cell center at time $t$.

Furthermore, the dimensionless inertial and aerodynamic torques of the wing are calculated as the sum of the cross product of each force and the positional vector at each cell center of the wing about the origin of body $\left(O_{b}\right)$, such that:

$$
\boldsymbol{T}_{\mathrm{iner}}^{*}=\sum_{i}\left(\boldsymbol{r}_{i}^{*} \times \boldsymbol{F}_{\mathrm{iner}, i}^{*}\right) \boldsymbol{T}_{\text {aero }}^{*}=\sum_{i}\left(\boldsymbol{r}_{i}^{*} \times \boldsymbol{F}_{\text {aero }, i}^{*}\right),
$$


where $\boldsymbol{r}_{i}^{*}$ denotes the dimensionless positional vector of the $i$-th cell center on the wing surface, and $\boldsymbol{F}_{\text {aero, } i}$ and $\boldsymbol{F}^{*}{ }_{\text {iner }, i}$ are the aerodynamic and inertial forces at the $i$-th cell center, respectively.

The aerodynamic and inertial powers are calculated as the scalar products of the velocity and the aerodynamic and inertial forces acting upon the wing as

$$
\boldsymbol{P}_{\text {iner }}^{*}=\sum_{i}\left(\boldsymbol{F}_{\text {iner, },}^{*} \cdot \boldsymbol{v}_{i}^{*}\right) \boldsymbol{P}_{\text {aero }}^{*}=\sum_{i}\left(\boldsymbol{F}_{\text {aero }, i}^{*} \cdot \boldsymbol{v}_{i}^{*}\right)
$$

Furthermore, the muscle-mass-specific aerodynamic and inertial powers as well as the total mechanical power $\boldsymbol{P}_{\text {total }}$ can be calculated as

$$
\boldsymbol{P}_{\text {iner }}=\boldsymbol{P}_{\text {iner }}^{*} / M_{\mathrm{m}}, \boldsymbol{P}_{\text {aero }}=\boldsymbol{P}_{\text {aero }}^{*} / M_{\mathrm{m}} \rightarrow \boldsymbol{P}_{\text {total }}=\boldsymbol{P}_{\text {iner }}+\boldsymbol{P}_{\text {aero }},
$$

where the mass of flight muscle, $M_{\mathrm{m}}$ is assumed to contribute to $30 \%$ of the total body mass [7].

\section{Verification and validation}

Verification and validation of the present multi-blocked, overset-grid NS solver are conducted through a variety of benchmark tests. Verification of the methods and the codes is first undertaken with a specific focus on its self-consistency in terms of the grid dependence and the time step effects. Validation of simulations of flapping flights is then executed in four fold: 1) whether the realistic geometric and kinematic models for hawkmoth, honeybee, and fruitfly in hovering are able to generate sufficient lifts to support the insect weights; 2) how well the multi-blocked, overset-grid, and fortified NS solver predicts the flapping-wing aerodynamics at high Reynolds numbers $(\mathrm{Re}=30000)$ in terms of time-varying thrusts generated by a purely plunging wing [33]; 3) how well the lift and drag forces predicted at lower Reynolds numbers $(\mathrm{Re}=234)$ acting upon a fruitfly wing in an idealized flapping mode match the measurements by a dynamically scaled fly-robot [11]; and 4) proof of capability in modeling realistic hawkmoth hovering in terms of near-and far-field vortex dynamics, wake structures, and flapping flight energetics involving force-generation and power requirements.

\section{A. Self-consistency and verification}

An extensive study on the grid dependence is conducted for hawkmoth hovering at $R e=6300$ and $k=0.3$, 
and for fruitfly hovering at $R e=134$ and $k=0.21$. For the hawkmoth hovering, we tested five cases: case 1 (wing grid: $45 \times 45 \times 31$, body grid: $33 \times 35 \times 35$ ), case 2 (wing grid: $45 \times 45 \times 31$, body grid: $45 \times 47 \times 65$ ), case 3 (wing grid: $45 \times 45 \times 31$, body grid: $45 \times 47 \times 95$ ), and case 4 (wing grid: $45 \times 45 \times 45$, body grid: $45 \times 47 \times 95$ ) all with a time step of $d t=0.01$, and case 5 (wing grid: $45 \times 45 \times 31$, body grid: $45 \times 47 \times 65$ ) with a time step of $d t=0.005$. The pseudo-compressibility coefficient $\lambda$ is set to be 1.0 for all the cases. The plotted in Fig. 5(a), 5(b) indicates that while a slight difference is visible in the case 1 almost no distinguished discrepancy is observed among the other four cases. Note that even in the case 1 the mean lift and drag coefficients are rather reasonable with a difference of less than $5 \%$ compared with the other cases. Results of the two time steps of 0.01 and 0.005 also show seldom difference and hence a time step of 0.01 is used for all the other simulations. For the fruitfly hovering, three cases are investigated of case 1 (wing grid: $45 \times 45 \times 31$, body grid: $33 \times 35 \times 35$ ) and case 2 (wing grid: $45 \times 45 \times 31$, body grid: $45 \times 47 \times 65$ ) at a time step of $d t=0.01$, and case 3 (wing grid: $45 \times 45 \times 31$, body grid: $45 \times 47 \times 65$ ) with a time step of $d t=0.005$. Again, the three force components (lift, drag, and sideslip force coefficient) as plotted in Fig. 5(c) obviously show seldom difference among the three cases.

\section{B. Comparison with measurements and validation}

\section{Comparisons of mean lift and drag forces}

Here the validation of insect hovering flights is achieved through comparisons of the mean lift and drag forces over a complete beat cycle in terms of sizing or Reynolds number effect. An extensive analysis of three typical hovering flights of the hawkmoth $(R e=6300)$, the honeybee $(R e=1134)$ and the fruitfly $(R e=134)$ is performed by using the realistic morphology and kinematics models as defined in the preceding sections. As shown in Fig. 6, the mean lift forces are calculated to be $17.0 \mathrm{mN}$ for the hawkmoth large enough to support a weight of $14.7 \mathrm{mN}, 7.60 \mathrm{mN}$ for the honeybee of a weight $6.86 \mathrm{mN}$, and $9.60 \times 10^{-3} \mathrm{mN}$ for the fruitfly of a weight $9.41 \times 10^{-3} \mathrm{mN}$, respectively. Moreover, the horizontal forces in the three hovering models are calculated to be less than $5 \%$ of the corresponding lift forces, obviously a negligible margin, which further validates the current hovering simulations. 
Validation of flapping wing aerodynamics at high Reynolds number

Validation of flapping wing aerodynamics is firstly implemented by an extended study on time accuracy of thrust forces generated by a rectangular wing oscillating in pure heave [33] (Fig. 7(a)). The wing is a $100 \mathrm{~mm}$ chord, $600 \mathrm{~mm}$ span, NACA0012 airfoil with an aspect ratio of 6 , plunging with constant amplitude $\left(h_{B A S E}=0.175 c\right.$, chord length $\left.c=100 \mathrm{~mm}\right)$ in a uniform flow $\left(U_{0}=0.393 \mathrm{~m} / \mathrm{s}\right)$. The displacement of the wing base is given by $s=h_{B A S E} \cos (\omega t)$ and the displacement of the wing tip is given by $s=h_{T I P} \cos (\omega t+\phi)$, where, for a rigid wing, $h_{T I P}=h_{B A S E}$ and the phase difference between the wing base and tip is 0 ; but for an inflexible wing as defined by [33] it was measured of $h_{T I P}=1.13 h_{B A S E}$ with a phase difference of $-4.2 \mathrm{deg}$. The Reynolds number is defined as $R e=U_{0} c / v=30000$, where $v$ is dynamic viscosity of water; the reduced frequency is defined as $k=\pi f c / U_{0}=1.82$, where $f$ is oscillating frequency. The thrust coefficient is defined by $C_{T}=2 T / \rho U_{0} c$ where $\rho$ is water density and $T$ is the thrust per unit span. This case is executed to validate the unsteady aerodynamics of flapping wings at high Reynolds numbers over a range of large insect flight (i.e. hawkmoth at $R e=6000$ ) and bird flight (e.g., hummingbird at $R e=6000 \sim 10000$ and bat at $R e>10000$ ).

Four cases are tested. The grid refinement is tested by using two single O-O type grids: a coarse grid $(35 \times 45 \times 65)$ with an eighteen chord large computational domain and a minimum grid spacing adjacent to the wing surface of $0.1 / \sqrt{ } \operatorname{Re} \approx 0.001$; and a fine grid $(45 \times 51 \times 91)$ with the same large domain and the minimum grid spacing. To verify time accuracy of the overset grid methods and the fortified NS solutions, a two-block grid system is further introduced, involving a wing-fitted grid $(35 \times 45 \times 27)$ of a two chord large domain and a global grid $(45 \times 51 \times 91)$. Effect of the wing flexibility in terms of spanwise bending on the thrust generation is also investigated using the fine single grid. The time step is taken as $d t=0.01$ for all the cases and the pseudo-compressibility coefficient $\lambda$ is set to be 1.0. Computed thrust coefficients are compared with the experimental results by Heathcote [33] and plotted in Fig. 7(b). It is seen that all the four cases show very reasonable agreement with the measured thrust coefficients. Results of the two-blocked overset grid match closely those of the fine grid, which hence verifies the effectiveness of the present fortified NS solver for the multi-blocked overset grid. The inflexible wing model shows closer agreement with the measurements in amplitude and phase, which indicates that the flexibility as observed in the experiments [33] is though very 
small but responsible for the slight increase in the thrust forces and results in a slight phase delay.

\section{Validation of flapping wing aerodynamics at low Reynolds number}

Furthermore, in order to validate hovering aerodynamics at low Reynolds numbers (i.e., for fruitfly or tiny insects), an extended study is further conducted for a fruitfly model in terms of time-varying lift and drag forces, which undergoes an idealized flapping-wing kinematics as defined by $[11,24]$. The realistic wing-body model of the fruitfly as shown in Fig. 2 is employed but with slight modifications for the wing model by adding a connecting portion at the wing base, which is identical to the mechanical wing model of the robo-fly [11]. The robo-fly wing model has a wing length of $L_{0}=24 \mathrm{~cm}$ and a mean wing-chord length, $c_{m}=10.42 \mathrm{~cm}$, resulting in a wing area of $S_{w}=167.0 \mathrm{~cm}^{2}$ and an aspect ratio of $A R=L_{0}{ }^{2} / S_{w}=3.74$. Since a connecting portion between the pivot point and the model wing base is approximately $3.75 \mathrm{~cm}\left(L_{d}=0.36 c_{m}\right)$, a resultant wing length (between the pivot point and the wing tip) $L=L_{0}+L_{d}=28.75 \mathrm{~cm}$. Note that the real wing length is important because it determines the wing tip velocity during flapping movements and accordingly the force generation. The wing kinematics is redefined based on those in [11,24], where a beat stroke as depicted in Fig. 8(a) consists of three parts: the pitching-down and translational acceleration at early stroke; the constant-speed translation and constant AoA during mid-stroke; and the pitching-up rotation and translational deceleration at late stroke. The translational velocities, the positional angles, the rotational angular velocities, and the AoAs are thereby described mathematically as:

1) Early stroke

$$
\begin{aligned}
& u_{t}^{+}=\sin \left(\pi \frac{\tau}{\Delta \tau_{t}}\right) ; \phi(t)=\frac{\Phi}{2}-\left(T \frac{U}{L}\right) \frac{\Delta \tau_{t}^{+}}{\pi}\left[1-\cos \left(\pi \frac{\tau}{\Delta \tau_{t}}\right)\right] \rightarrow 0<\tau<\frac{\Delta \tau_{t}}{2}, \\
& \dot{\alpha}_{t}^{+}=-\dot{\alpha}_{0}^{+} \cos \left(\pi \frac{\tau-\tau_{r 0}}{\Delta \tau_{r}}\right) ; \alpha(t)=\frac{\pi}{2}-\alpha_{a} \sin \left(\pi \frac{\tau-\tau_{r 0}}{\Delta \tau_{r}}\right) \rightarrow 0<\tau<\tau_{r 0}+\frac{\Delta \tau_{r}}{2},
\end{aligned}
$$

2) Mid-half stroke

$$
\begin{aligned}
& u_{t}^{+}=1.0 ; \phi(t)=\frac{\Phi}{2}-\left(T \frac{U}{L}\right)\left[\frac{\Delta \tau_{t}^{+}}{\pi}+\left(\tau^{+}-\frac{\Delta \tau_{t}^{+}}{2}\right)\right] \rightarrow \frac{\Delta \tau_{t}}{2}<\tau<\tau_{1}, \\
& \dot{\alpha}_{t}^{+}=0 ; \alpha(t)=\frac{\pi}{2}-\alpha_{a} \rightarrow \tau_{r 0}+\frac{\Delta \tau_{r}}{2}<\tau<\tau_{r 0}+\frac{T-\Delta \tau_{r}}{2},
\end{aligned}
$$




\section{3) Half-end stroke}

$$
\begin{aligned}
& u_{t}^{+}=\cos \left(\pi \frac{\tau-\tau_{1}}{\Delta \tau_{t}}\right) ; \phi(t)=\frac{\Phi}{2}-\left(T \frac{U}{L}\right)\left[\frac{\Delta \tau_{t}^{+}}{\pi}\left(1+\sin \left(\pi \frac{\tau-\tau_{1}}{\Delta \tau_{t}}\right)\right)+\left(\tau_{1}^{+}-\frac{\Delta \tau_{t}^{+}}{2}\right)\right] \rightarrow \tau_{1}<\tau<\tau_{1}+\Delta \tau_{t}, \\
& \dot{\alpha}_{t}^{+}=\dot{\alpha}_{0}^{+} \cos \left(\pi \frac{\tau-T / 2-\tau_{r 0}}{\Delta \tau_{r}}\right) ; \alpha(t)=\frac{\pi}{2}+\alpha_{a} \sin \left(\pi \frac{\tau-T / 2-\tau_{r 0}}{\Delta \tau_{r}}\right) \rightarrow \tau_{r 0}+\frac{T-\Delta \tau_{r}}{2}<\tau<\tau_{r 0}+\frac{T+\Delta \tau_{r}}{2},
\end{aligned}
$$

4) Mid-half stroke

$$
\begin{aligned}
& u_{t}^{+}=-1.0 ; \phi(t)=\frac{\Phi}{2}-\left(T \frac{U}{L}\right)\left[\frac{\Delta \tau_{t}^{+}}{\pi}+2 \tau_{1}^{+}-\left(\tau^{+}-\frac{\Delta \tau_{t}^{+}}{2}\right)\right] \rightarrow \tau_{1}+\Delta \tau_{t}<\tau<2 \tau_{1}+\frac{\Delta \tau_{t}}{2}, \\
& \dot{\alpha}_{t}^{+}=0 ; \alpha(t)=\frac{\pi}{2}+\alpha_{a} \rightarrow \tau_{r 0}+\frac{T+\Delta \tau_{r}}{2}<\tau<T+\tau_{r 0}-\frac{\Delta \tau_{r}}{2},
\end{aligned}
$$

5) End stroke

$$
\begin{aligned}
& u_{t}^{+}=\sin \left(\pi \frac{\tau-T}{\Delta \tau_{t}}\right) ; \phi(t)=\frac{\Phi}{2}-\left(T \frac{U}{L}\right) \frac{\Delta \tau_{t}^{+}}{\pi}\left(1-\cos \left(\pi \frac{\tau-T}{\Delta \tau_{t}}\right)\right) \rightarrow 2 \tau_{1}+\frac{\Delta \tau_{t}}{2}\left(T-\frac{\Delta \tau_{t}}{2}\right)<\tau<T, \\
& \dot{\alpha}_{t}^{+}=-\dot{\alpha}_{0}^{+} \cos \left(\pi \frac{\tau-T-\tau_{r 0}}{\Delta \tau_{r}}\right) ; \alpha(t)=\frac{\pi}{2}-\alpha_{a} \sin \left(\pi \frac{\tau-T-\tau_{r 0}}{\Delta \tau_{r}}\right) \rightarrow T+\tau_{r 0}-\frac{\Delta \tau_{r}}{2}<\tau<T .
\end{aligned}
$$

The translational velocity is denoted by $u_{t}^{+}$nondimensionally, which takes a constant value $\left(u_{t}^{+}=u_{t} / U=1.0\right.$ where $U$ is the reference velocity) except at pronation and supination when the wing rotates. $\Delta \tau_{t}$ denotes duration of the wing deceleration/acceleration whereas $\tau_{1}$ represents time instant when the deceleration at the end of the half stroke starts, which may be represented by $\tau_{t}=\left(T-\Delta \tau_{t}\right) / 2$. Note that there exists a specific relationship among the stroke amplitude, the reference velocity, the length and the period, such as:

$$
\frac{\Phi L}{U}=\frac{T}{2}-\Delta \tau_{t}\left(1-\frac{2}{\pi}\right)
$$

where the parameter $U T / L$ can be determined when the stroke amplitude $\Phi$ and $\Delta \tau_{t} / T$ are given. For instance, given a $\Phi=160 \mathrm{deg}(2.79 \mathrm{rad})$ and $\Delta \tau_{t} / T=0.16, U T / L$ is calculated to be 5.92 accordingly.

The rotational velocity is denoted by $\alpha_{t}^{+}$nondimensionally, and its amplitude $\dot{\alpha}_{0}^{+}$is a constant that can be determined when $\Delta \tau_{r}$ is given. The AoA amplitude $\alpha_{a}$ can be determined, e.g., when the wing rotates from $\alpha=0$ deg to $\alpha=140$ deg. $\Delta \tau_{r}$ denotes duration of the wing rotational phase whereas $\tau_{r 0}$ represents time 
instant when the wing starts rotational phase, which is identical to the three modes as defined in [11], of the advanced $\left(\tau_{r 0}<0\right)$, the symmetric $\left(\tau_{r 0}=0\right)$, and the delayed one $\left(\tau_{r 0}>0\right)$, respectively.

Based on the geometry and the flapping frequency $(145 \mathrm{mHz})$ of the model wing used by [11] as well as the density $\left(0.88 \times 10^{3} \mathrm{kgm}^{-3}\right)$ and the kinematic viscosity $(115 \mathrm{cSt})$, a mean velocity at the wing tip is calculated to be $U=0.26 \mathrm{~ms}^{-1}$, which, together with the reference length of the mean chord length, results in a Reynolds number of 234 and a reduced frequency of 0.3 . The rotational axis of the wing is fixed at a distance of $0.2 c_{m}$ from the leading edge.

Two models involving a wing-body model (wing grid: $45 \times 45 \times 31$, body grid: $33 \times 35 \times 35$ ) and a wing-wing model without the body (wing grid: $45 \times 45 \times 31$, no-body grid: $35 \times 45 \times 91$ ) are tested for the advanced mode $[11,24]\left(\Delta \tau_{r}=0.24, \tau_{r 0}<0=-0.1\right)$. Each computation is done for four stroke cycles and the time varying lift and drag coefficients are plotted for the fourth cycle as in Fig. 8(b) compared with the measurements [11]. Note that the lift and drag forces measured are also nondimensionalized using the reference length and velocity. Although a perfect match of the parameters $\Delta \tau_{t} / T, \Delta \tau_{r}$ and $\tau_{r 0}$ with those used in the experiment [11] is impossible and the two models are in reasonable good agreement with the measurements. The coarse grid model with the body shows lower values in both lift and drag forces and some phase differences. The fine grid model without the body is obviously in very close agreement in both peak values and phases. Note that the computed drag coefficient turns to be negative during the upstroke because a local wing-base-fixed coordinate system is used here which does not reverse during rotational phase. Interestingly, the two force peaks as detected and referred to be responsible for the rotational and wake-capture mechanisms [11] are observed corresponding to the transient phase with a high translational acceleration and a jump in rotational acceleration. This implies that the idealized flapping-wing kinematics somehow lead to an impulsive-start effect in terms of the rotational angular acceleration, which may, as a result, enlarge the rotational effects compared with the realistic insect flapping flights [31].

\section{Proof of capability: a case study of insect hovering flight}

So far we have shown that the present integrated modeling approach is robust and efficient in modeling 
realistic insect flapping flights through a wide range of Reynolds numbers from $O\left(10^{2}\right)$ to $O\left(10^{4}\right)$, covering most of the insect species. Next, by taking hawkmoth hovering as an instance, we show that this insect dynamic flight simulator is capable to integrate vortex dynamics and wake topology about flying insects, and to elucidate a correlation between the induced flows and the force production in insect flapping flights.

As of spatially and transiently complicated features, it is usually of difficulties but great importance to quantify and to visualize the near-field vortex dynamics in terms of leading edge, trailing edge and tip vortices, and the far-field wake topology including vortex shedding and break-up and downwash [28-31]. Here we approach this problem by introducing an effective means of combining the normalized helicity density $(h=V \bullet \omega /|V||\omega|)$ and the enstrophy density $\left(\varepsilon=|\omega|^{2} / 2\right)$ in identifying and extracting the vortical structures. Here $V$ and $\omega$ denote velocity and vortical vectors, respectively. Note that the normalized helicity density $h$ defines as the projection of a fluid's spin vector in the direction of its momentum vector, being positive (red) if it points in the same direction and negative (blue) if it points in the opposite direction. This method, by the enstrophy density, identifies and frames a vortical structure as the region of large vorticity; and, by the normalized helicity density, visualizes simultaneously the helical feature of the vortical structure on its iso-surface.

\section{A. Near-and far-field flow: vortex dynamics and wake structures}

Vortex dynamics in the near field is illustrated in Fig. 9 where, as marked on the time-varying flapping angles in Fig. 4(b), sequence of four iso-surface plots of $\varepsilon$ and $h$ is presented to visualize the formation and development of the vortical structures in the hawkmoth hovering during pronation (A), downstroke (B), supination (C) and upstroke (D). Iso-surfaces of the enstrophy density are used to visualize the vorticity: (A) $\varepsilon=1.2$; (B) $\varepsilon=1.4$; (C) $\varepsilon=1.2$; and (D) $\varepsilon=0.85$; the color mapping of the normalized helicity density varies between -1 and +1 .

At the early downstroke when the wing proceeds to late pronation undergoing a pitch-down rotation ((A) Fig. 4(b)), a horseshoe vortex (HSV) is observed wrapping each wing, which is formed from a leading-edge vortex (LEV), a wing tip vortex (TV), and a trailing-edge vortex (TEV) (Fig. 9(A). The LEV 
and TEV grow in size and in strength, stretching from the wing tip towards the wing base; the TEV subsequently detaches from the wing, forming the starting vortex but connecting to the TV. Eventually, the HSVs including the attached LEVs, the enlarged TVs and the detached TEVs grow to a downstroke vortex tube ring (DVTR) pair (Fig. 9(B)), which forms two closed-loops wrapping the wing pair. This DVTR pair creates two three-dimensional irregular vortex sheets through the half-stroke corresponding to changes in circulation of the bound vorticity around the two wings, which roll up and deform under the influence of their own induced velocity fields. The shed TEV shows a helical structure with an intense vortical core and moves forward with the wing, which results in a VTR core enclosing an area much less than the vortex sheet area; and the downstroke vorticity produced by the wings are concentrated around the perimeter of the DVTR pair, forming the hovering downwash (Fig. 10(a)).

During the upstroke (Fig. 9(C), 9(D)) similar vortical structures are observed: a TV generated in the late half of supination during a pitch-up rotation of wing ((C) in Fig. 4(b)) grows up into an upstroke HSV forming the UVTR pair; the UVTR also forms a three-dimensionally skewed vortex sheet with roll-ups and a downward jet through its core (Fig. 10(b)). Compared with the DVTR the UVTR pair shows an elongated sheet and hence a larger core probably because it has a free shed TEV without attachment onto the body which may lower the strength and the stability of its vorticity (Fig. 9(D)).

Fig. 10(a), 10(b) illustrates the downwash wake topology in terms of top and front views at down-and up-stroke. Iso-velocity surfaces are visualized with a magnitude of $v_{z}=-0.5$ in perspective views while velocity contours and vectors are in top and front views. Note that the vortex tube ring pair in a fashion of iso-surface is superimposed onto the velocity contours and vectors, which illustrates a clear image of two intense jets present in the cores of the vortices forming the hovering downwash.

The front views (Fig. 10(a), 10(b)) show vertically pulsed lattice downwash wakes, synonymous with the shedding and diffusion of the VTR wake at down-and up-stroke; top views further reveal that the cores of the momentum jets (or, the jet columns) oscillate horizontally. The hawkmoth hovering shows two largely distorted momentum jet columns, which, in fact, link together, resulting in a pronounced wider downwash disc and hence a relatively narrower low-speed zone right below the body. The downwash wakes show high 
induced-velocity areas at the VTR cores (over a range of $0.5 \sim 0.9 \mathrm{U}_{\text {ref }}$ ), which is comparable to the mean wing tip speed and is dependent upon flapping amplitude and wing morphology. In addition, it is interesting to find that the conventional downwash contraction is not observed in the present computations, which states that the downwash wake contracts and is accelerated when it diffuses downward $[4,14,18]$.

\section{B. Energetics: forces and powers}

Plots in Fig. 5(a), 5(b) show the time courses of lift and drag forces in a complete beat cycle. $T$ denotes dimensionless period of one flapping cycle. Compared with low Reynolds number flapping flight, e.g., fruitfly hovering [31], two peaks in lift are observed here in the high Reynolds number case at upstroke and at downstroke, which depends upon the flapping wing kinematics and, as a result, the LEVs and VTRs are very likely responsible for these.

Furthermore, based on the computed instantaneous aerodynamic forces and the wing velocities, the instantaneous muscle-mass-specific inertial, aerodynamic and mechanical powers are calculated and plotted in Fig. 11. The muscle-mass-specific aerodynamic power $P_{\text {aero }}$ is the power required to overcome air resistance (Eq. (44)). $P_{\text {aero }}$ reaches a maximum value in the late phase of both the downstroke and the upstroke. During the translational phase, the insect undergoes costly aerodynamic power of the wings due to high-aerodynamic force production. The computed mean aerodynamic power $\left(90 \mathrm{Wkg}^{-1}\right)$ is approximately the same with the previous experimental results [28]. The muscle-mass-specific inertial power $P_{\text {iner }}$ is the power needed to accelerate the mass of the wing (Eq. (44)), which shows an increase as the wing accelerates, and decreases when the wing decelerates. Note that the negative sign of $P_{\text {iner }}$ means that the direction of the forces acting on the wing is opposite to that of the wing velocity. The $P_{\text {iner }}$ exhibits negative value in the most part of the downstroke and the upstroke except for the wing acceleration phase. The computed mean inertial power required to accelerate the wing amounts to $65 \mathrm{Wkg}^{-1}$. Note that we assume that the wing deceleration accrues with no cost and there is no elastic storage.

Total muscle-mass-specific mechanical power, $P_{\text {total }}$, is the mechanical powers required to move the wings. It is calculated by summing $P_{\text {iner }}$ and $P_{\text {aero }}$ (Eq. (44)). Peaks of $P_{\text {total }}$ appear in the early phase of the down-and up-strokes. The $P_{\text {total }}$ becomes negative in the late upstroke as the decelerating wings produce less 
power than is required to overcome the inertial forces. The computed time course of $P_{\text {total }}$ is in qualitative agreement with the experimental results [28].

\section{Summary}

We have developed a robust and efficient computational approach for modeling insect flapping flights. This model is featured by the integrated modeling of realistic body-wing morphology, realistic flapping-wing and body kinematics, and unsteady aerodynamics based on a multi-blocked, overset grid, fortified NS solver. We chose three typical insects of hawkmoth, honeybee and frutifly, which have a representative body length from $5 \mathrm{~cm}$ (moth) down to $3 \mathrm{~mm}$ (fly) corresponding to a wide range of Res $O\left(10^{2}\right)$ to $O\left(10^{4}\right)$, and construct three realistic morphological and kinematics models. We validate these insect hovering flights through a variety of benchmark tests on the self-consistency and verification of the method and the code, the quantitative comparison of force production and energetics with reliable experimental data, and the visualization of the near-and far-field vortical structures about the hovering flyers. Our results demonstrate that the present model, as an insect dynamic flight simulator, is of high efficiency in accurately modeling and quantitatively evaluating insect flapping flights.

Further improvements to the model will be the incorporation of the solutions to the equation of the 6DOF motion involving the flapping wings and the body, and a coupling between the dynamics of the wing-body motion and the unsteady aerodynamics based on the NS equations. These changes, if achieved, will enable the simulation of free-flying insects and simultaneously the establishment of a design tool for micro air vehicles.

\section{Acknowledgments}

This work is partly supported by a PRESTO-JST program, and the Grant-in-Aid for Scientific Research of No. 18656056 and No. 18100002, JSPS, Japan. The computations were performed on a supercomputer at the Information Center, the Institute of Physical and Chemical Research (RIKEN), Japan.

\section{References}


[1] M. J. Lighthill, Mathematical Biofluid-dynamics. Philadephia: SIAM (1975).

[2] T. Weis-Fogh. Energetics of hovering flight in hummingbirds and drosophila. J. Exp. Biol. 56, 79 (1972).

[3] C.P. Ellington, The aerodynamics of hovering insect flight. VI. Lift and power requirements. Phil. Trans. R. Soc. Land B 305, 115 (1984).

[4] C.J. Pennycuick, Bird Flight Performance, A Practical Calculation Manual, Oxford University Press (1989).

[5] K. Fujii, Unified zonal method based on the fortified solution algorithm. J. Comput. Phys. 118, 92 (1995).

[6] C. von Den Berg, C.P. Ellington, The vortex wake of a 'hovering' model hawk moth. Phil. Trans. R. Soc. Land B 352, 317 (1997).

[7] F. O. Lehmann, M. H. Dickinsn, The changes in power requirements and muscle efficiency during elevated force production in the fruit fly Drosophila melanogaster. J. Exp. Biol. 200, 1133 (1997).

[8] C.P. Ellington, C. Van Den Berg, A. P. Willmott, A.L.R. Thomas, Leading-edge vortices in insect flight. Nature 384, 626 (1998).

[9] H. Liu, C.P. Ellington, K. Kawachi, Coen van den Berg, A.P. Willmott, A computational fluid dynamic study of hawkmoth hovering. J. Exp. Biol. 201, 461 (1998).

[10] H. Liu, K. Kawachi, A Numerical Study of Insect Flight. J. Comput. Phys. 146 (1), 124 (1998).

[11] M. H. Dickinson, F. O. Lehmann, S. P. Sane, Wing rotation and the aerodynamic basis of insect flight. Science 284, 1954 (1999).

[12] N.C. Prewitt, D.M. Belk, W. Shyy, Parallel computing of overset grids for aerodynamic problems with moving objects. Progress in Aerospace Sciences, 36, 117 (2000).

[13] J. M. Birch, M. H. Dickinson, Spanwise flow and the attachment of the leading-edge vortex on insect wings. Nature 412, 722 (2001).

[14] M. Sun, J. Tang, Unsteady aerodynamic force generation by a model fruit-fly wing. J. Exp. Biol. 205, 55 (2002). 
[15] R.B. Srygley, A.L.R. Thomas, Unconventional lift-generating mechanisms in free-flying butterflies. Nature 420, 660 (2002).

[16] S.N. Fry, R. Sayaman, M. H. Dickinson, The aerodynamics of free-flight maneuvers in Drosophila. Science, 300 (18), 495 (2003).

[17] D.R. Warrick, B.W. Tobalske, D.R. Powers, Aerodynamics of the hovering hummingbird. Nature 435, $1094(2005)$.

[18] Z.J. Wang, Dissecting insect flight. Ann. Rev. Fluid Mech. 37, 183 (2005).

[19] L.A. Miller, C. S. Peskin, A computational fluid dynamics of 'clap and fling' in the smallest insects. J. Exp. Biol. 208, 195 (2005).

[20] H. Liu, Simulation-Based Biological Fluid Dynamics in Animal Locomotion. Transaction of the ASME Applied Mechanics Reviews, 58, 269 (2005).

[21] D.L. Altshuler, W.B. Dickson, J.T. Vance, S.P. Roberts, M.H. Dickinson, Short-amplitude high-frequency wing strokes determine the aerodynamics of honeybee flight. PNAS, 102, 18213 (2005).

[22] S.N. Fry, M.H. Dickinson, The aerodynamics of hovering flight in Drosophila. J. Exp. Biol. 208, 2303 (2005).

[23] F-O, Lehman, S.P. Sane, M.H. Dickinson, The aerodynamic effects of wing-wing interaction in flapping insect wings. J. Exp. Biol. 208, 3075 (2005).

[24] A. Gilmanov, F. Sotiropoulos, A hybrid Cartesian/immersed boundary method for simulating flows with 3D geometrically complex moving bodies. J. Comput. Phys. 207, 457 (2005).

[25] S.P. Sane, N.P. Jacobson, Induced airflow in flying insects II. Measurement of induced flow. J. Exp. Biol. 209, 43 (2006).

[26] C. Poelma, W.B. Dickson, M.H. Dickinson, Time resolved reconstruction of the full velocity field around a dynamically scaled flapping wing. Expts Fluids, 41, 213 (2006).

[27] H. Dong, R. Mittal, F.M. Najjar, Wake Topology and Hydrodynamic Performance of Low-Aspect-Ratio Flapping airfoil. J. Fluid Mech. 566, 309 (2006).

[28] R.J. Bomphrey, G.K. Taylor, N.J. Lawson, A.L.R. Thomas, Digital particle image velocimetry measurements of the downwash distribution of a desert locust Schistocerca gregaria.. J. R. Soc. Interface 3, 
311 (2006).

[29] R. Ramamurti, W.C. Sandberg, A computational investigation of the three-dimensional unsteady aerodynamics of Drosophila hovering and maneuvering. J. Exp. Biol. 210, 881 (2007).

[30] A. Hedenstrom, L.C. Johansson, M. Wolf, R. von Busse, Y. Winter, G.R. Spedding, Bat Flight Generates Complex Aerodynamic Tracks. Science 316, 894 (2007).

[31] H. Aono, F. Liang, H. Liu, Near-and far-field aerodynamics in hovering insects: an integrated computational study. J. Exp. Biol. 211, 239 (2007).

[32] H. Liu (unpublished)

[33] S. Heathcote, Z. Wang, and I. Gursul, Effects of spanwise flexibility on flapping wing propulsion, J. Fluids and Structures, (2007). 


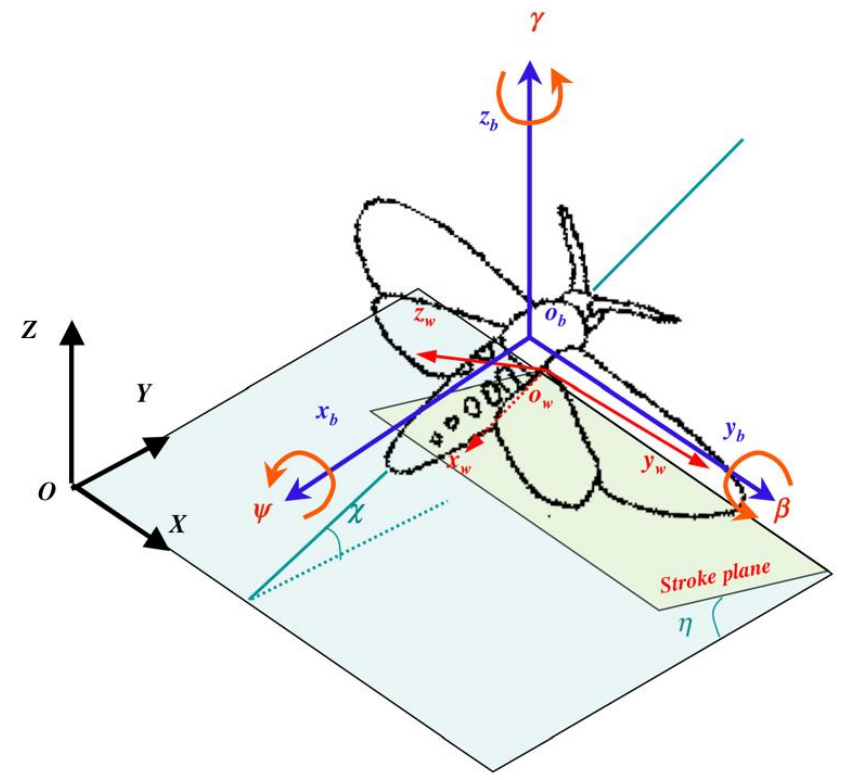

(a)

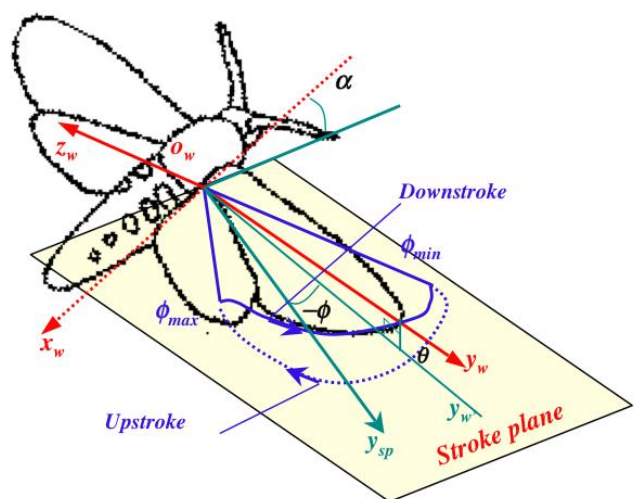

(b)

Fig. 1 Definitions (a) of the wing-fixed system $\left(x_{w}, y_{w}, z_{w}\right)$, the body-fixed system $\left(x_{b}, y_{b}, z_{b}\right)$, and the global system $(X, Y, Z)$; the stroke plane angle $\eta$ and the body angle $\chi$, the angles of pitch $\beta$, roll $\psi$, and yaw $\gamma$ with respect to the body-fixed system. (b) Wing position parameters within the stroke plane: the wingtip path, the positional angle $\phi$, the elevation angle $\theta$, and the angle of attack $\alpha$. 

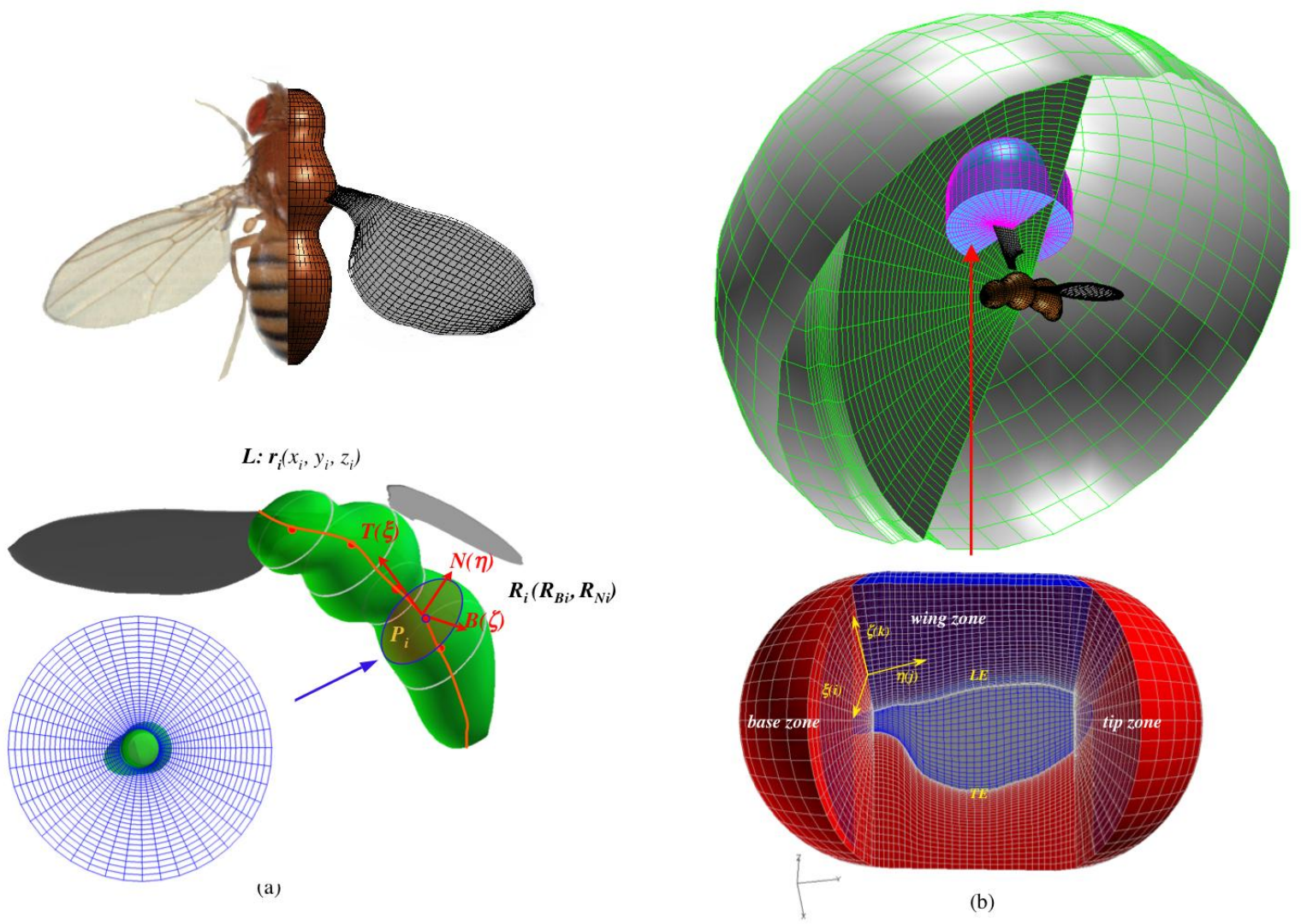

Fig. 2 Schematic diagram of an image-and differential geometry-based method for morphological modeling and a multi-block model for insect-like flyers. (a) A top-view fruit fly with a computer model superimposed on the right half and a perspective view of the model with the extracted body centerline $L$ and elliptic cross sections. (b) A three-block model and grid systems for the fruit fly with two wing-fitted blocks immersed within a body-fitted block. 


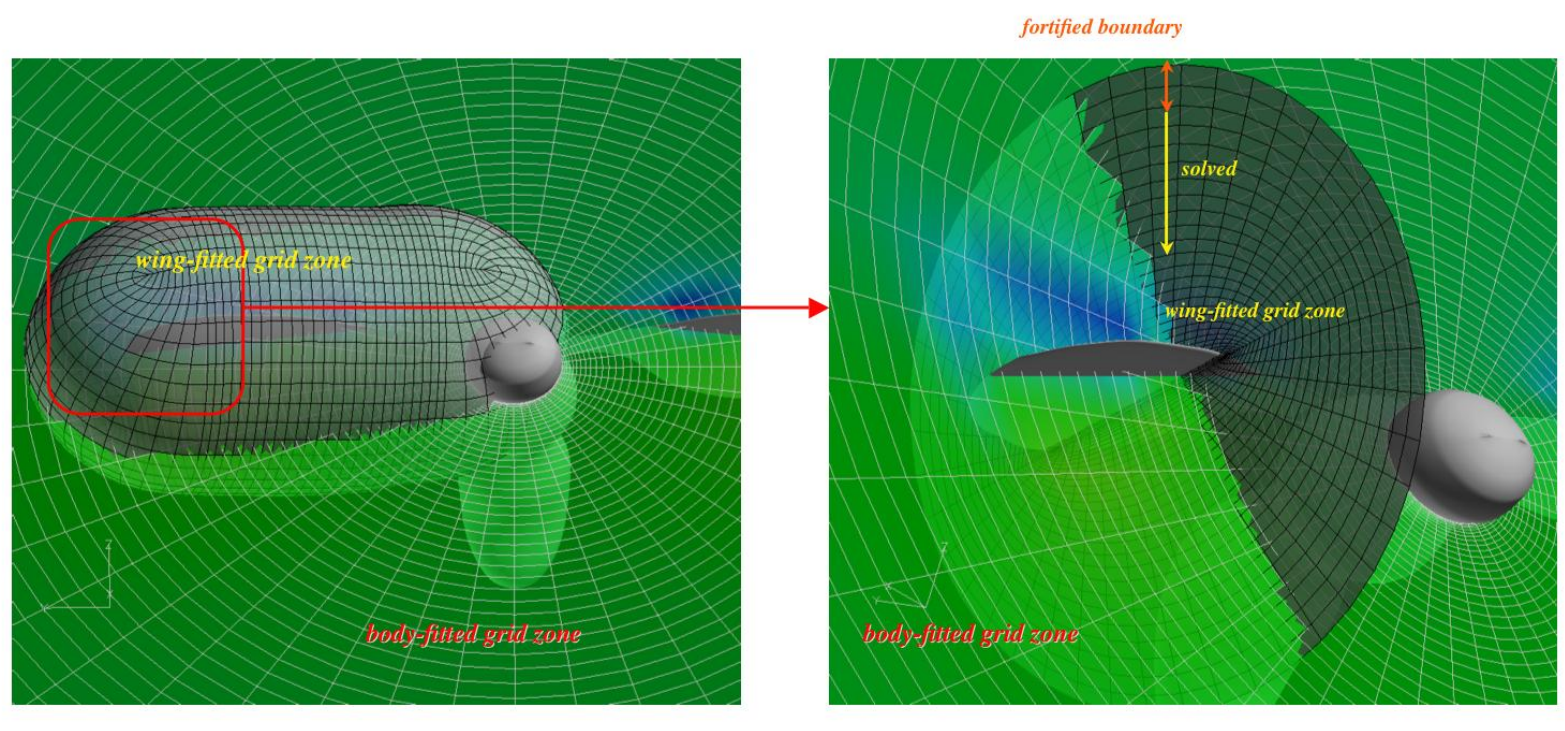

(a)

(b)

Fig. 3 (a) Overlapping grids with holes cut and (b) fortified solution zonal procedure for the overlaid grid. 


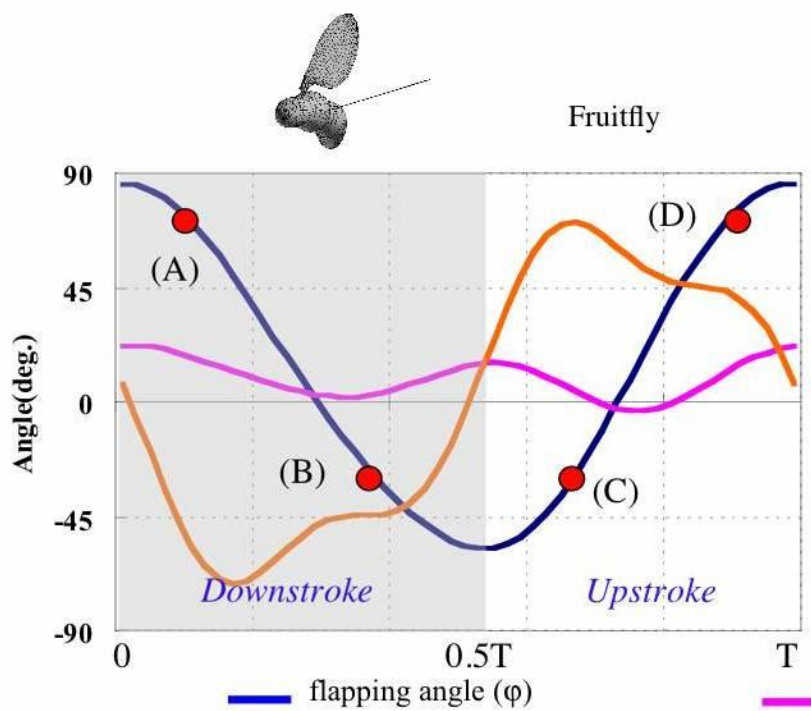

(a)

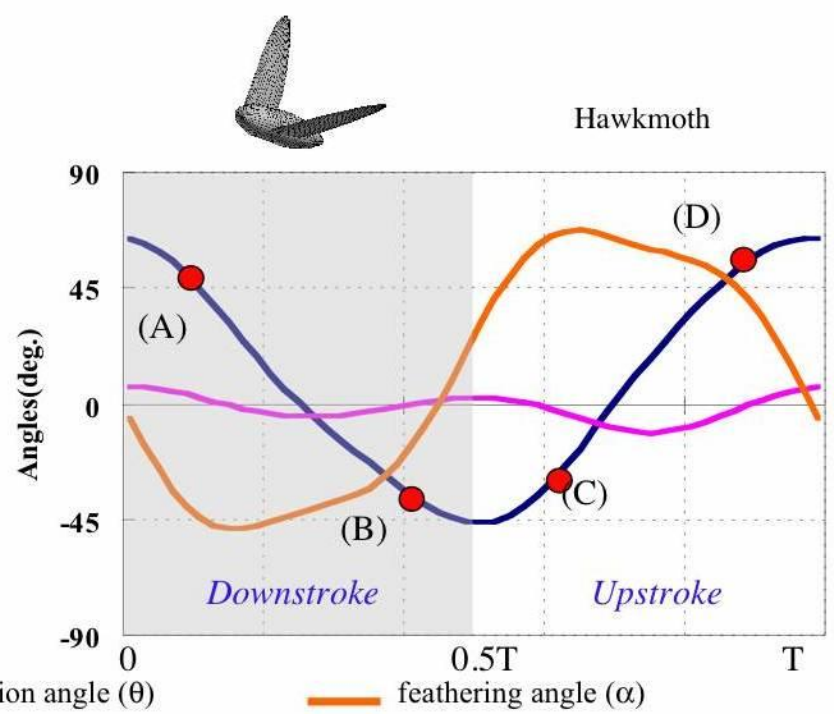

(b)

Fig. 4 Kinematic models in terms of time course of flapping, elevation and feathering angles: (a) a fruit fly: (b) a hawkmoth. 
(a)

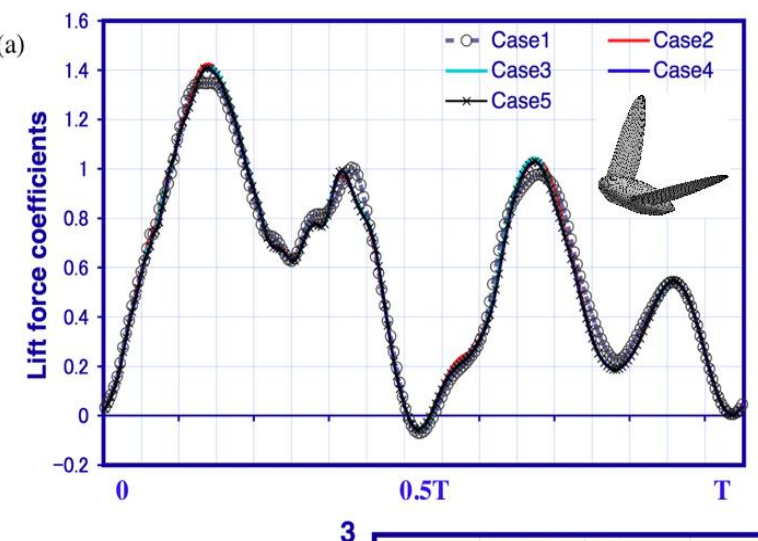

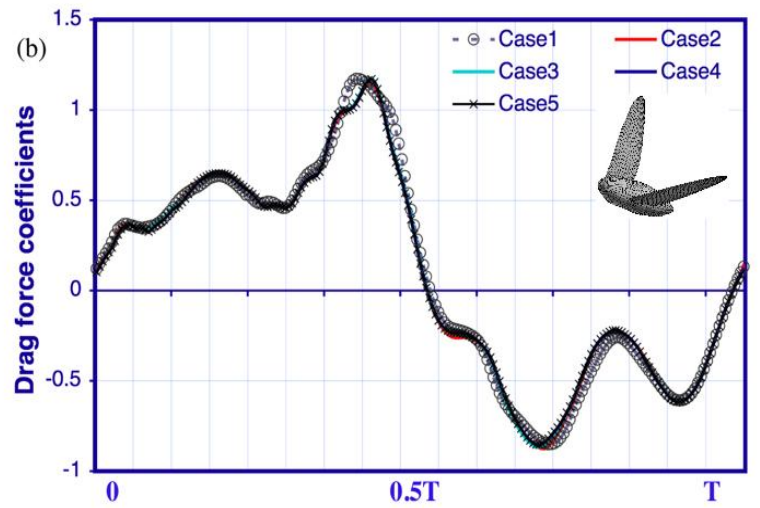

(c)

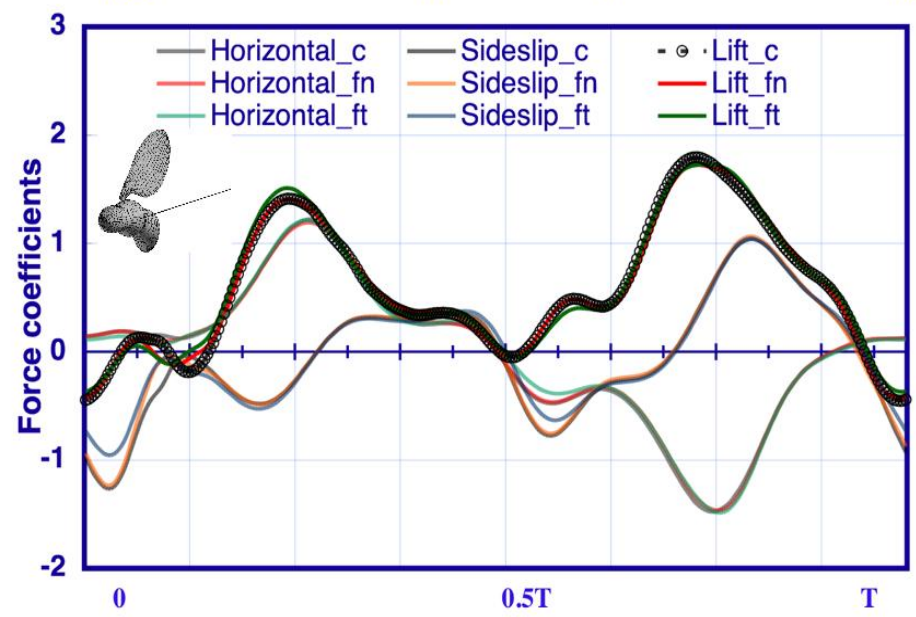

Fig. 5 Effects of grid and time step on time courses of lift and drag force coefficients over a stroke cycle. (a) Lift and (b) drag coefficients in hawkmoth hovering in five cases. (c) Lift, drag and sideslip force coefficients in fruitfly hovering in three cases. 


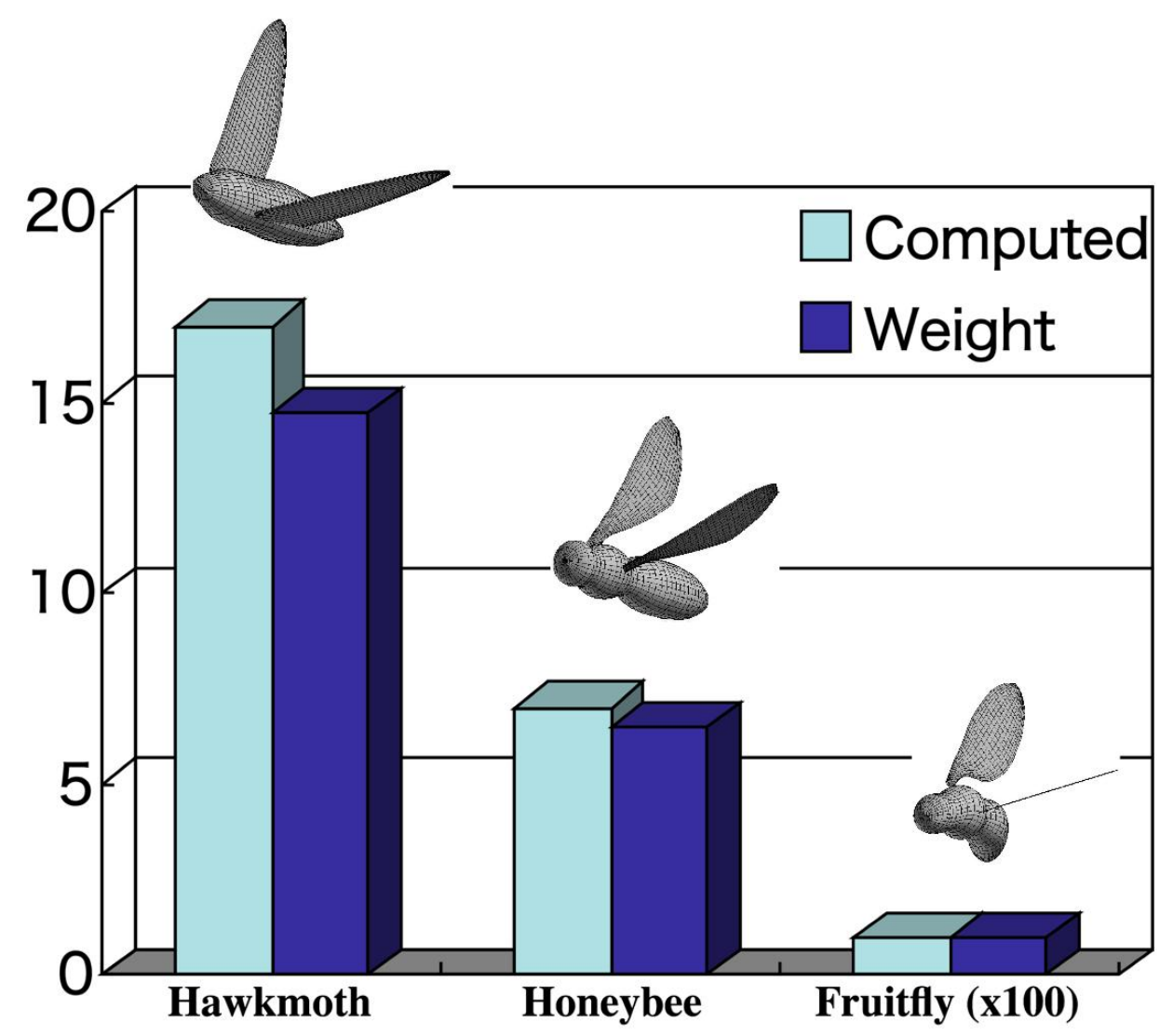

Fig. 6 Weights and computed lift forces $(m N)$ in hovering flights of hawkmoth, honeybee and fruitfly. 
(a)

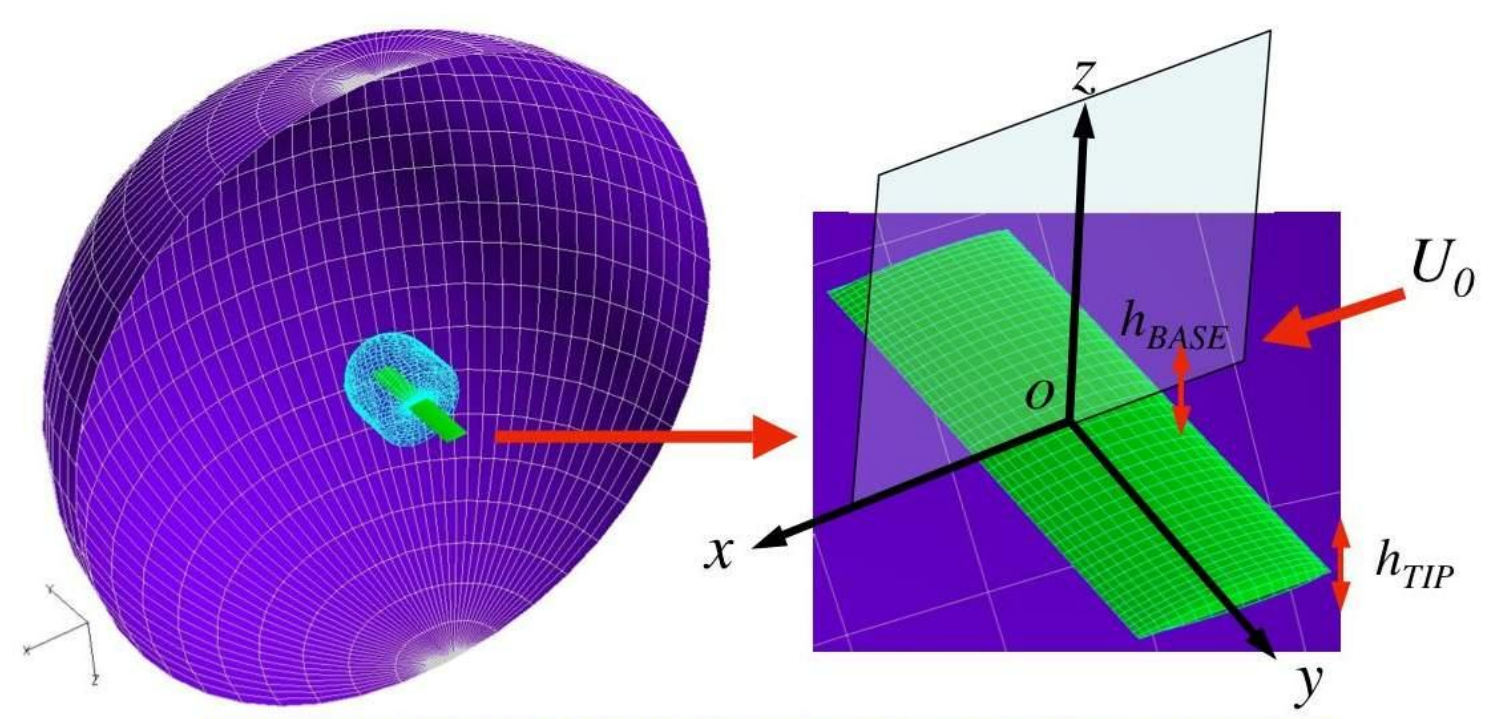

(b)

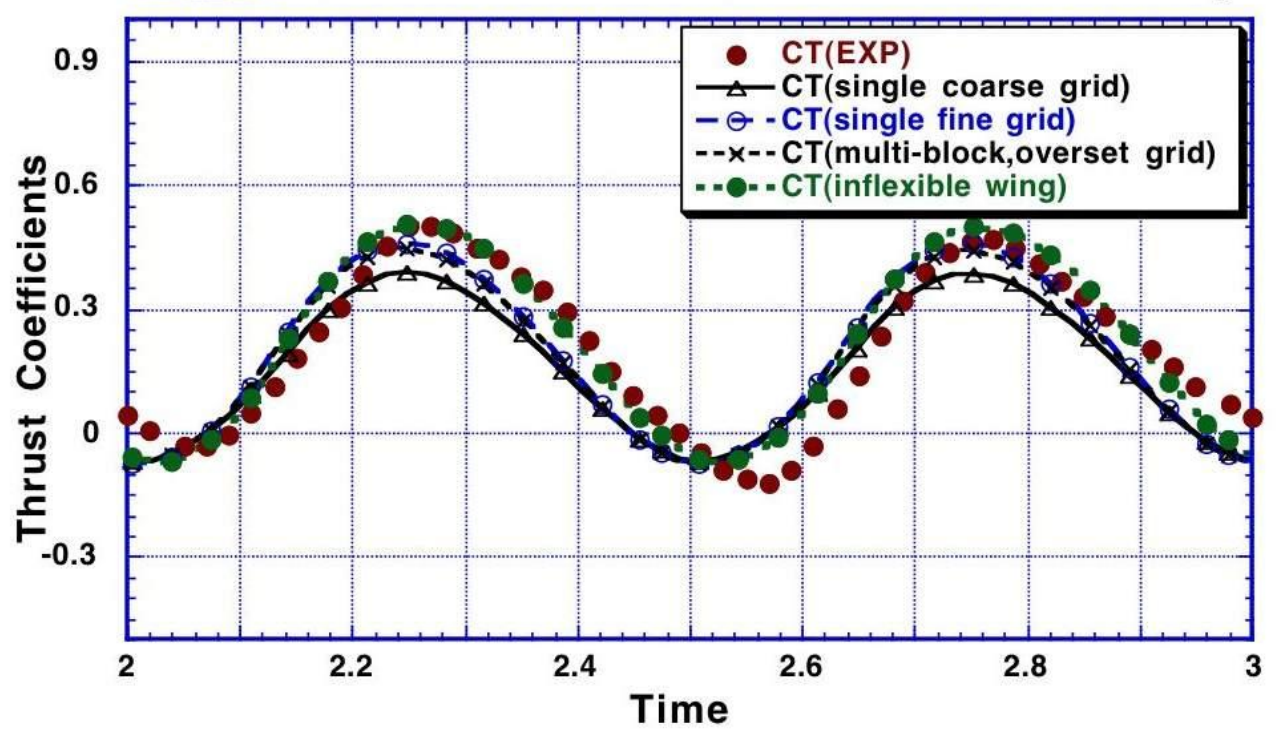

Fig. 7 (a) Two-blocked overset grids around a NACA0012 wing in pure heave. (b) Time course of thrust coefficients compared with measurements [33]. 
(a)
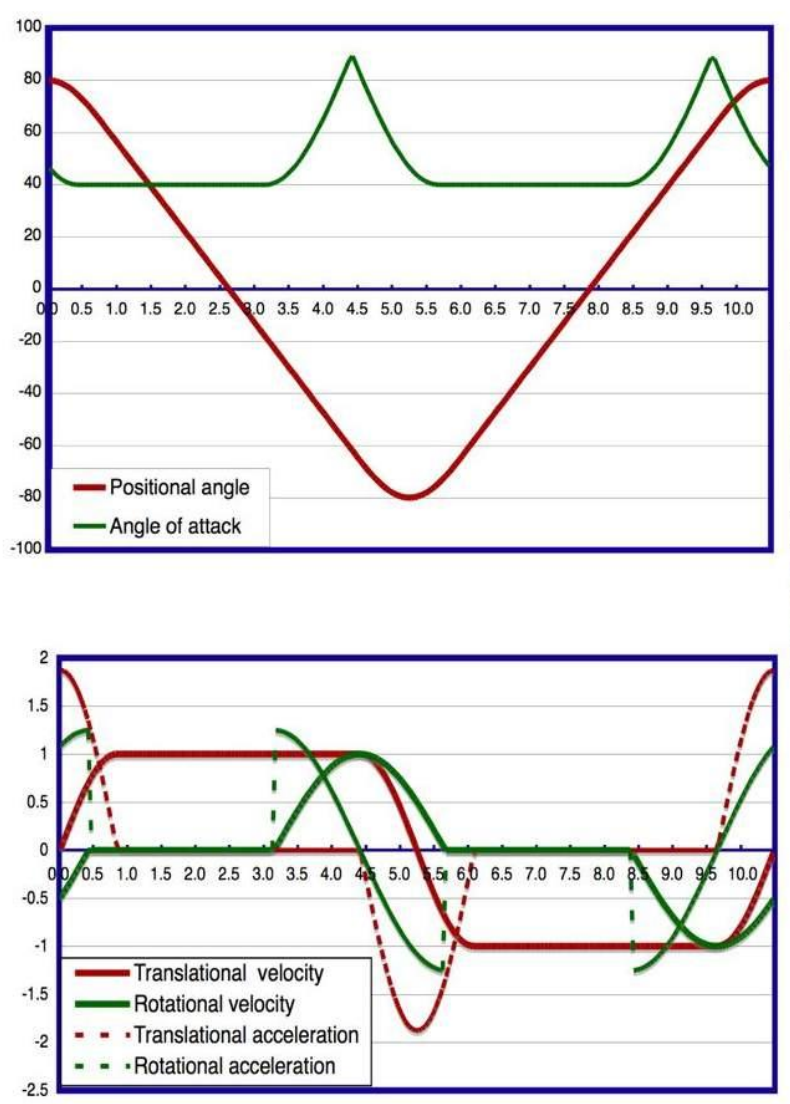

(b)

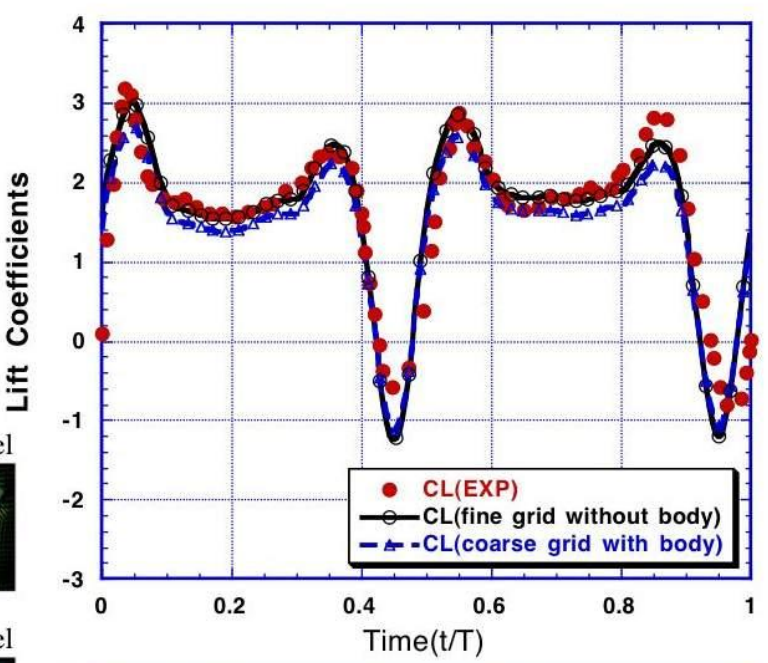

Wing-wing model

Wing-body model
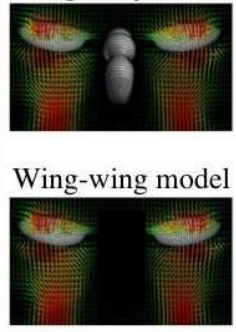

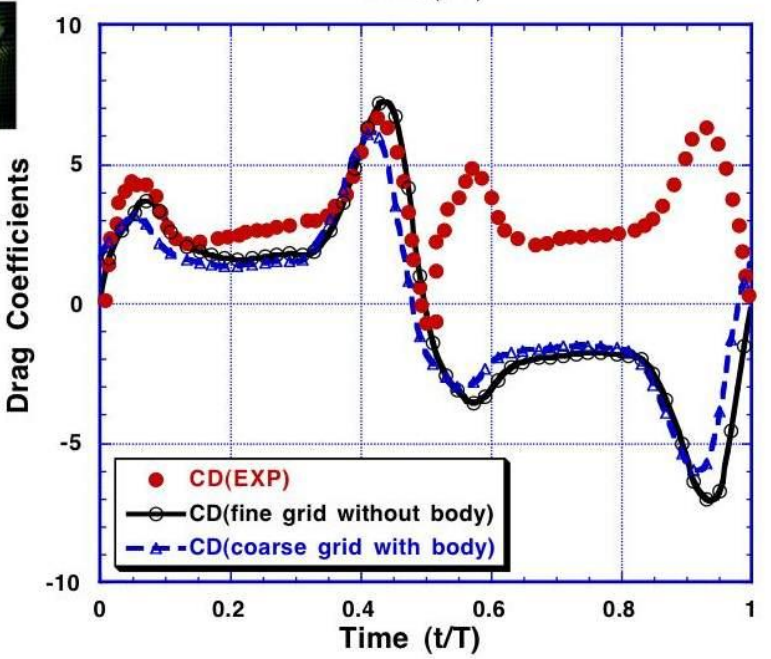

Fig. 8 (a) Idealized wing movements of translation and rotation (angles, velocities and accelerations). (b)

Time course of lift and drag coefficients compared with measurements [11]. 

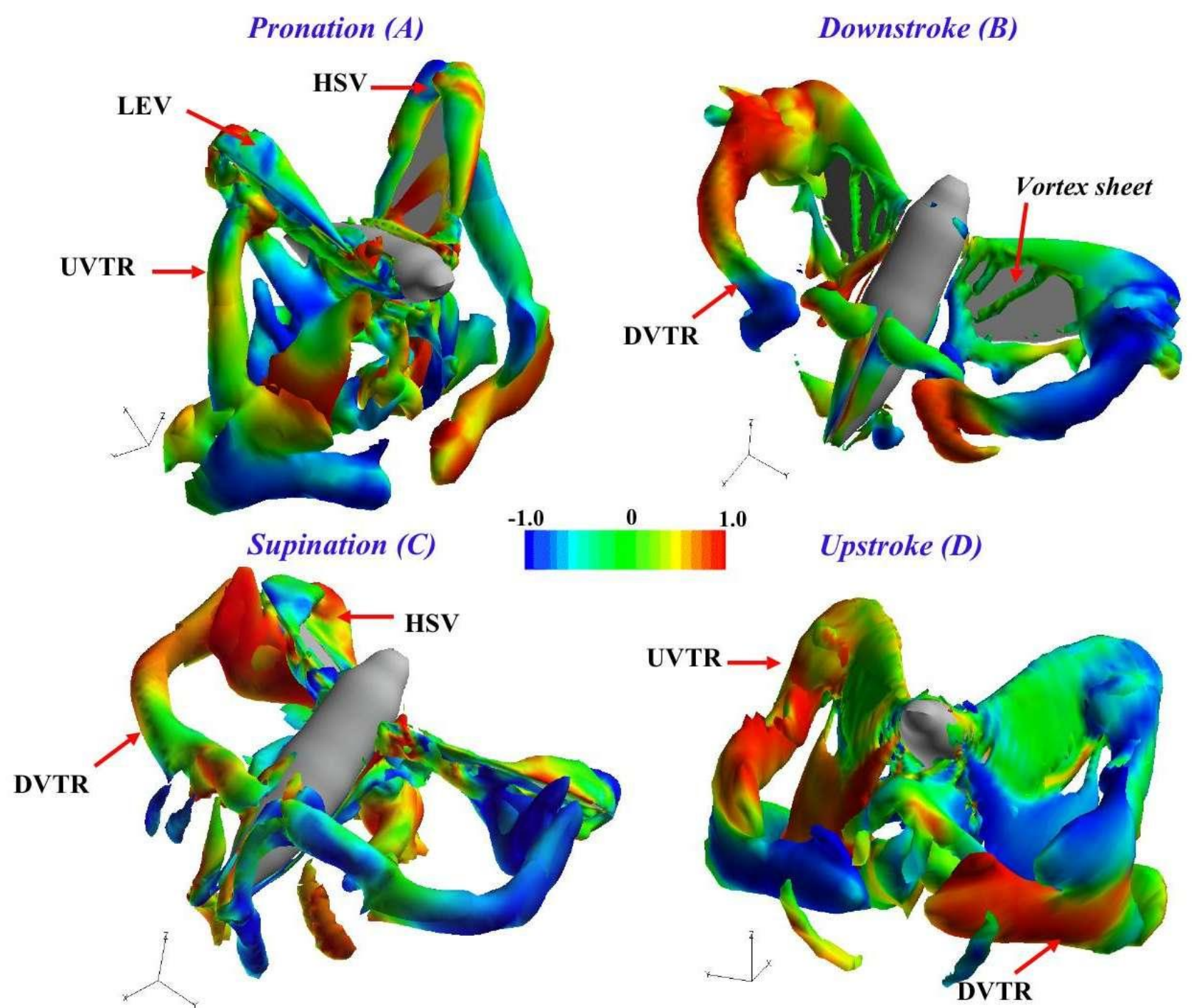

Fig. 9 Near-and far-field vortical flow structures about a hovering hawkmoth model $(\operatorname{Re}=6300, k=0.3)$. 

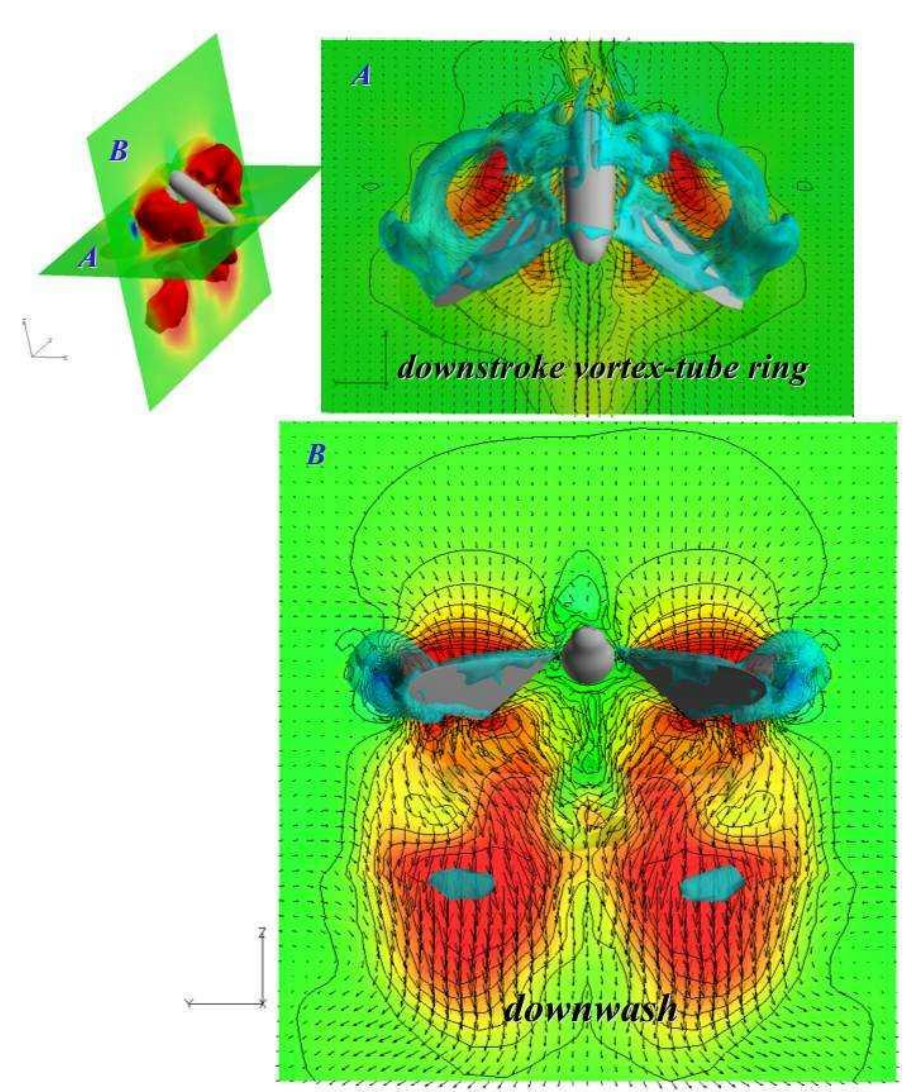

(a) Downstroke

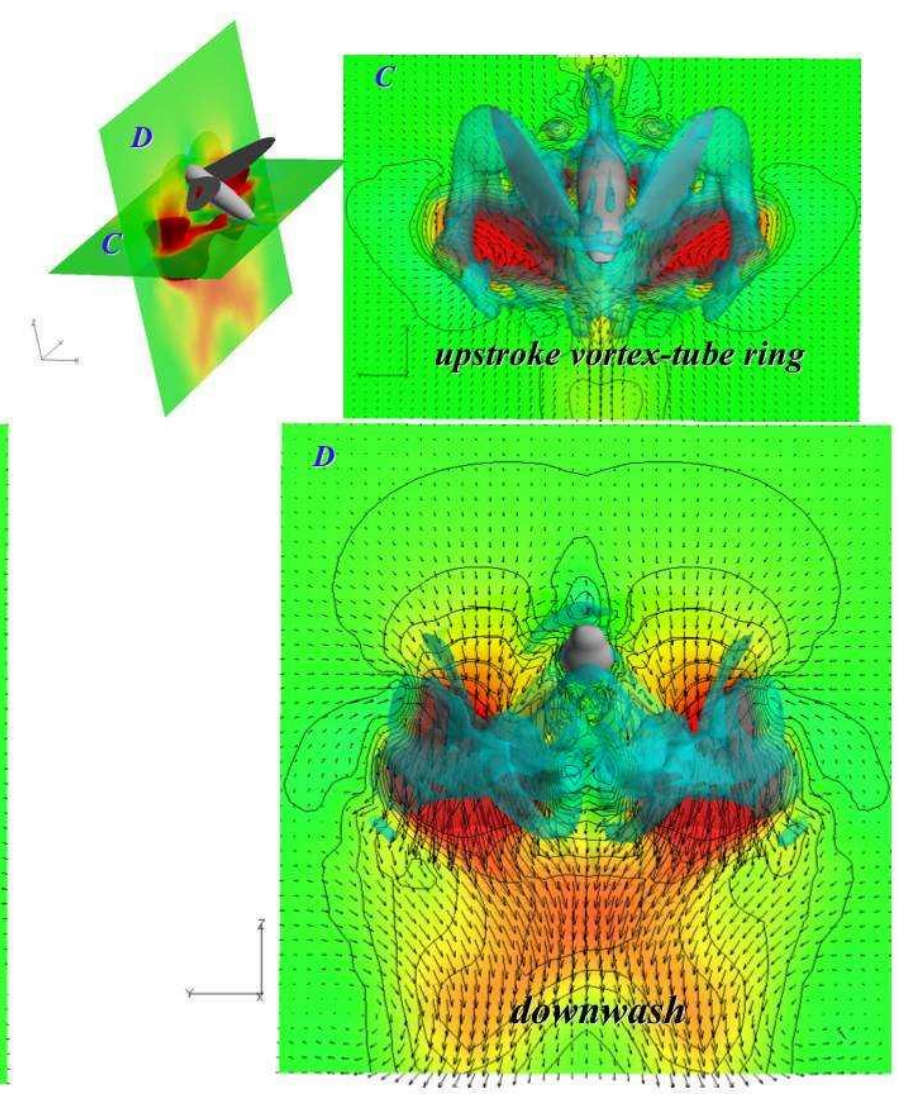

(b) Upstroke

Fig. 10 Wake topology and downwash about a hovering hawkmoth model $(\mathrm{Re}=6300, \mathrm{k}=0.3)$. 


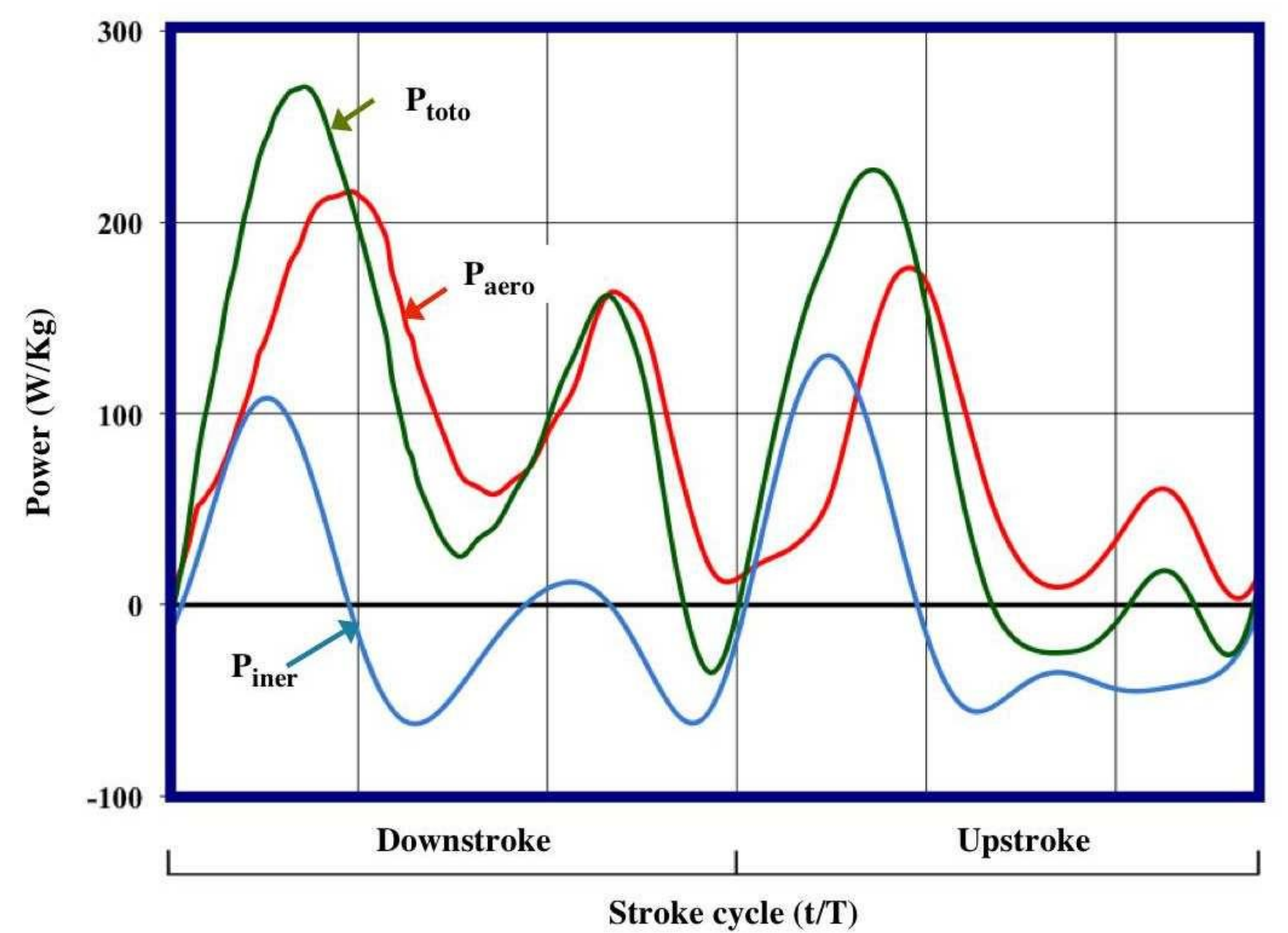

Fig. 11 Time courses of muscle-mass-specific powers: inertial powers $\left(\mathrm{P}_{\text {iner }}\right)$, aerodynamic powers $\left(\mathrm{P}_{\text {aero }}\right)$ and total powers $\left(\mathrm{P}_{\text {toto }}\right)$. 